\title{
A necessary and sufficient instability condition for inviscid shear flow
}

\author{
N. J. Balmforth \\ and \\ P. J. Morrison * \\ February 1998
}

\begin{abstract}
We derive a condition that is necessary and sufficient for the instability of inviscid, twodimensional, plane parallel, shear flow with equilibrium velocity profiles that are monotonic, real analytic, functions of the cross stream coordinate. The analysis, which is based upon the Nyquist method, includes a means for delineating the possible kinds of bifurcations that involve the presence of the continuous spectrum, including those that occur at nonzero wavenumber. Several examples are given.
\end{abstract}

Key words: Shear flow, stability theory, Nyquist method.

\section{Introduction}

The linear stability of inviscid, incompressible, two-dimensional, plane parallel, shear flow was considered over a century ago by Rayleigh, Kelvin, and others. A principal result on the subject is Rayleigh's celebrated inflection point theorem [1], which states that for an equilibrium flow to be unstable, the equilibrium velocity profile must contain an inflection point. That is, if the velocity profile is given by $U(y)$, where $y$ is the cross-stream coordinate, then there must be a point, $y=y_{I}$, for which $U^{\prime \prime}\left(y_{I}\right)=0$. Much later, in 1950, Fjørtoft [2] generalized the theorem by showing that, moreover, if there is one inflection point, then $U^{\prime \prime \prime}\left(y_{I}\right) / U^{\prime}\left(y_{I}\right)<0$ is required for instability (see [3] for further extensions). Both Rayleigh's Theorem and Fjørtoft's subsequent generalization are necessary conditions for instability, but they are not sufficient. That is, even though an equilibrium profile may contain a vorticity minimum, it is not necessarily unstable. The point of this paper is to derive, for a large class of equilibrium velocity profiles, a condition that is necessary and sufficient for instability.

\subsection{Overview}

The procedure we use to derive the instability condition is inspired by techniques developed for the Vlasov equation. For that problem one can find a condition that is necessary and sufficient

*Address for correspondence: Department of Physics, C1600, University of Texas, Austin, TX 78712 
for instability by using the Nyquist method, a method that leads to what is known as the Penrose criterion [4] in plasma physics. The Penrose criterion follows fairly straightforwardly for the linear Vlasov problem because the discrete eigenvalues satisfy an explicit dispersion relation. However, in the context of the Euler equation governing the shearing fluid, the relevant eigenvalue problem leads to Rayleigh's equation. This equation cannot be manipulated into an explicit dispersion relation, and a sufficient condition for instability of velocity profiles of a general form has not previously been given. We note, though, that [5] and [6] present a sufficient condition for long wave instability, and in [5] a necessary and sufficient condition for instability of profiles with a single inflection point (where bifurcations are restricted to occur through zero wavenumber) was obtained.

Here we derive a condition that is necessary and sufficient for instability for a class of velocity profiles, $U(y)$, where $y$ is the cross stream coordinate. Specifically, we consider profiles that are monotonic functions when $y \in[-1,1]$, which we refer to as the "flow domain," and which are real analytic. This latter limitation means that $U(y)$ has a convergent Taylor series on $[-1,1]$ and thus possesses an analytic continuation into the complex $y$-plane. Hence, there exists a neighborhood (an open set of the complex plane) that contains the interval $[-1,1]$ in which $U(y)$ is analytic and in which $U^{\prime}(y) \neq 0$. We assume this neighborhood is as large as is needed in subsequent calculations. Both the monotonicity and analyticity limitations can be generalized, but we will not attempt this here.

\subsection{Summary of result}

In the remainder of this section we summarize our main result, which amounts to a prescription for obtaining the condition that is necessary and sufficient for instability: given the solution, $\psi\left(y, c_{r}\right)$, to the Fredholm integral equation,

$$
\psi\left(y, c_{r}\right)=\mathcal{G}\left(y, y_{c}\right)+\int_{-1}^{1} \frac{\mathcal{G}\left(y, y^{\prime}\right)-\mathcal{G}\left(y, y_{c}\right)}{U\left(y^{\prime}\right)-c_{r}} U^{\prime \prime}\left(y^{\prime}\right) \psi\left(y^{\prime}, c_{r}\right) d y^{\prime},
$$

where $c_{r}=U\left(y_{c}\right)$ and $\mathcal{G}\left(y, y^{\prime}\right)$ is a Green function (given in Eq. 8 below) containing the streamwise wavenumber $k$ and embodying the boundary conditions, we construct the "Nyquist function,"

$$
\epsilon\left(c_{r}\right)=1-\mathcal{P} \int_{-1}^{1} \frac{U^{\prime \prime}(y) \psi\left(y, c_{r}\right)}{U(y)-c_{r}} d y-i \pi \frac{U^{\prime \prime}\left(y_{c}\right) \psi\left(y_{c}, c_{r}\right)}{U^{\prime}\left(y_{c}\right)},
$$

where $\mathcal{P}$ denotes the Cauchy principal value. We then plot $\epsilon\left(c_{r}\right)$ on the $\left(\epsilon_{r}, \epsilon_{i}\right)$-plane for $c_{r}$ running from $U(-1)$ to $U(1)$, or, equivalently, for $y_{c}$ along the interval $[-1,1]$. The profile $U(y)$ is exponentially unstable for that value of $k$ used in Eq. (1) if and only if the resulting path loops around the origin, $\epsilon_{r}=\epsilon_{i}=0$. In fact, the path can only cross the $\epsilon_{r}$-axis at the inflection points, $y_{I}$, of the velocity profile where $U^{\prime \prime}\left(y_{I}\right)=0$. This leads to the following necessary and sufficient condition for instability: $\epsilon_{r}\left(c_{I}\right)<0$ with $c_{I}=U\left(y_{I}\right)$, or equivalently

$$
\int_{-1}^{1} \frac{U^{\prime \prime}(y) \psi\left(y, c_{I}\right)}{U(y)-c_{I}} d y>1
$$

for one of the $y_{I}$ 's.

In Section 7, among other examples, we treat the profile $U(y)=\tanh (\beta y)$ and arrive at the "Nyquist plots" shown in Fig. 1. For this equilibrium profile with $\beta=2$ the plots of Fig. 1(a) loop around the origin and $\epsilon_{r}\left(c_{I}\right)<0$ (where $c_{I}=0$ ) provided $k \lesssim 1.832$. Hence for $\beta=2$ the 

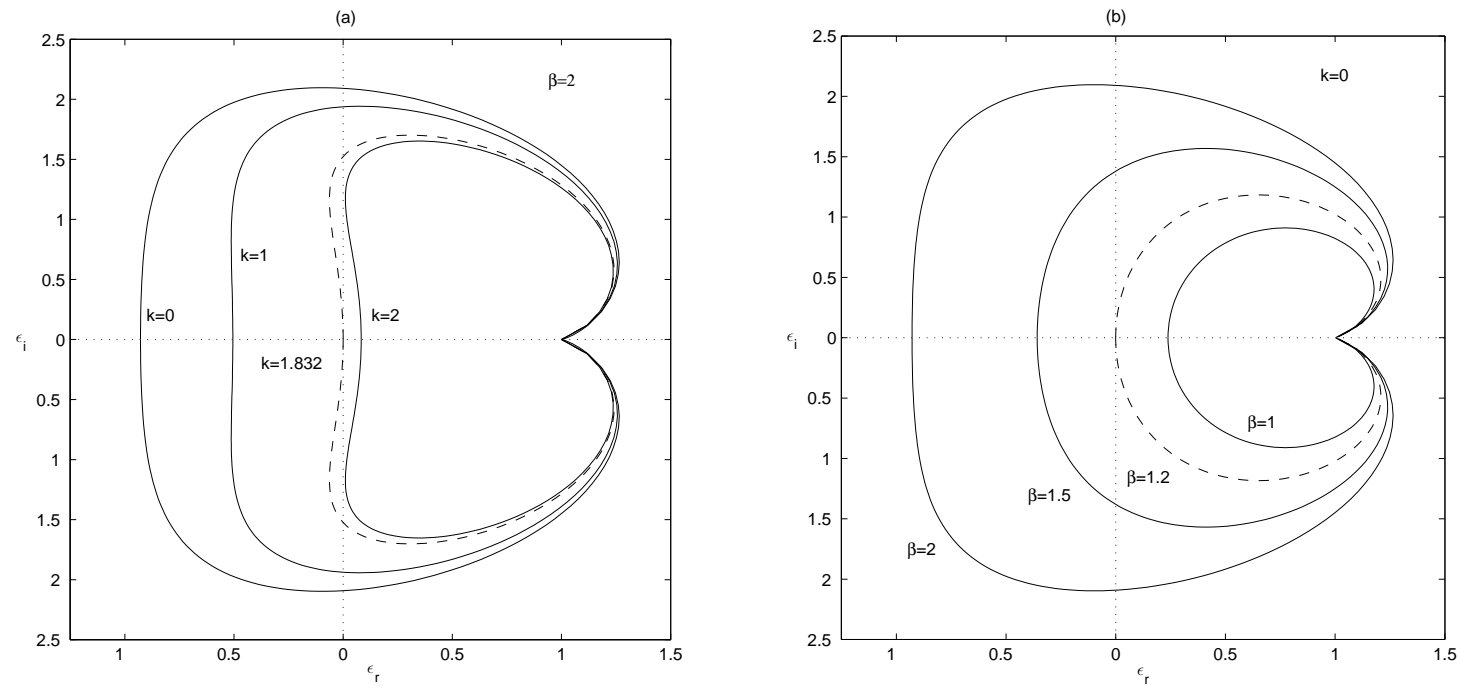

Figure 1: Nyquist plots for the single inflection point profile, $U(y)=\tanh \beta y$. (a) Four plots for $\beta=2$ and $k=0,1$, and 2 , and the critical value for the onset of instability, $k=k_{c} \simeq 1.832$ (dashed curve). (b) Four plots for $k=0$ and $\beta=1,1.5$, and 2, and the critical value for the onset of instability, $\beta=\beta_{c} \simeq 1.2$ (dashed curve).

profile is unstable over the band of wavenumbers $0<k \lesssim 1.832$. Figure 1 (b) illustrates the onset of instability, which occurs through $k=0$, as the parameter $\beta$ is changed.

In order to prove the result outlined above we need various mathematical results concerning Rayleigh's equation. These are derived in Sections 2-5. Application to specific equilibrium velocity profiles is presented in Sections 6 and 7. Finally, in Section 8 we summarize, place our work in context, and discuss future developments.

\section{Review}

\subsection{Formulation}

For a two-dimensional, inviscid fluid contained within the channel, $x \in(-\infty, \infty)$ and $y \in[-1,1]$, an equilibrium state is given by any flow, $(U, 0)$, where $U=U(y)$ is the equilibrium velocity profile. Infinitesimal disturbances to such an equilibrium are described by the equation [1],

$$
\partial_{t} \tilde{\omega}+U(y) \partial_{x} \tilde{\omega}=U^{\prime \prime}(y) \partial_{x} \tilde{\psi}
$$

where $\tilde{\omega}(x, y, t)$ is the vorticity perturbation, $\tilde{\psi}(x, y, t)$ is the associated streamfunction, which are related by

$$
\tilde{\omega}=\nabla^{2} \tilde{\psi}
$$

and the boundary conditions are $\tilde{\psi}(x, \pm 1, t)=0$. Throughout this paper we will consider solutions of the form $\tilde{\omega}=\omega(k, y, t) \exp (i k x)$ and $\tilde{\psi}=\psi(k, y, t) \exp (i k x)$, in which case (4) becomes

$$
\partial_{t} \omega+i k U(y) \omega=i k U^{\prime \prime}(y) \psi,
$$


and the inverse of (5) takes the form,

$$
\psi(y, t)=\int_{-1}^{1} \mathcal{G}\left(y, y^{\prime}\right) \omega\left(y^{\prime}, t\right) d y^{\prime}
$$

where

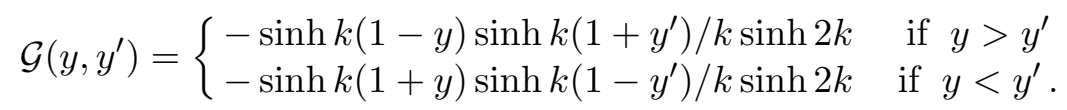

Here and henceforth we suppress the $k$-dependence in the arguments of all functions.

\subsection{Laplace transform}

We need some results associated with the Laplace transform approach to the solution of (6) as an initial value problem; so we give a brief review of this approach here. More detailed accounts are given in [7], [8], and with considerably more rigor in [9].

Recall, the Laplace transform pair is

$$
\Psi(y, p)=\int_{0}^{\infty} e^{-p t} \psi(y, t) d t, \quad \psi(y, t)=\frac{1}{2 \pi i} \int_{C} e^{p t} \Psi(y, p) d p
$$

where $C$ is the Bromwich contour that runs parallel to the imaginary $p$-axis and to the right of all singularities of the integrand. Using the above, (6) becomes

$$
\left(\frac{\partial^{2}}{\partial y^{2}}-k^{2}-\frac{i k U^{\prime \prime}}{p+i k U}\right) \Psi(y, p)=\frac{\omega(y, 0)}{p+i k U},
$$

where $\omega(y, 0)$, the initial value of the vorticity, satisfies $\omega(y, 0)=\left(\partial^{2} / \partial y^{2}-k^{2}\right) \psi(y, 0)$. The solution to (8) can be written formally as

$$
\Psi(y, p)=\int_{-1}^{1} \mathcal{H}\left(y, y^{\prime} ; p\right) \frac{\omega\left(y^{\prime}, 0\right)}{p+i k U\left(y^{\prime}\right)} d y^{\prime}
$$

where the Green function, $\mathcal{H}\left(y, y^{\prime} ; p\right)$, is given by

$$
\mathcal{H}\left(y, y^{\prime} ; p\right)= \begin{cases}-\Psi_{1}(y, p) \Psi_{2}\left(y^{\prime}, p\right) / W\left(\Psi_{1}, \Psi_{2}\right) & \text { if } \quad-1 \leq y \leq y^{\prime} \\ -\Psi_{1}\left(y^{\prime}, p\right) \Psi_{2}(y, p) / W\left(\Psi_{1}, \Psi_{2}\right) & \text { if } \quad y^{\prime} \leq y \leq 1\end{cases}
$$

with the Wronskian

$$
W\left(\Psi_{1}, \Psi_{2}\right):=\Psi_{1}(y, p) \Psi_{2}^{\prime}(y, p)-\Psi_{1}^{\prime}(y, p) \Psi_{2}(y, p) .
$$

Here $\Psi_{1}$ and $\Psi_{2}$ are any two solutions to (10) with the right-hand side set to zero and that satisfy the boundary conditions, $\Psi_{1}(-1, p)=\Psi_{2}(1, p)=0$. Since (10) does not contain a term involving the first derivative with respect to $y$, it is an elementary result (see [10], Chap. V) that the Wronskian is independent of $y$. Arguments pertaining to the inverse Laplace transform of (11) imply that the dispersion relation for discrete normal modes is given by

$$
W\left(\Psi_{1}, \Psi_{2}\right)=0,
$$

with $\operatorname{Re}(p)>0$. 


\section{$2.3 \quad$ Normal modes}

An alternative approach to Laplace transformation follows when we search for normal modes at the outset by assuming that $\omega(y, t)=\omega(y) \exp (-i k c t)$ and $\psi(y, t)=\psi(y) \exp (-i k c t)$. Inserting these expressions into (6) yields Rayleigh's equation,

$$
(U-c) \omega=(U-c)\left(\psi^{\prime \prime}-k^{2} \psi\right)=U^{\prime \prime} \psi .
$$

which is an equation for the eigenfunction $\psi(y)$ corresponding to the eigenvalue $c$. In the next section we describe some properties of this equation, for both real and imaginary values of $c$, that we will need later.

\section{Rayleigh's equation}

\subsection{Singular points and solutions in the complex plane}

We will need several facts about the solutions to Rayleigh's equation (15). Firstly, the point $y_{c}$, for which $U\left(y_{c}\right)=c$, is a singular point of (15). If $c$ is real then this point is commonly referred to as a "critical layer." Since $U(y)$ is a monotonic function for $y \in[-1,1]$ there is at most one such critical layer in the flow domain, and this occurs when $c$ is in the range of $U(y)$. Because of monotonicity and real analyticity of $U$ for $y$ in the flow domain, there exists a neighborhood of $[-1,1]$ in the complex plane in which the only singular point of (15) is the critical layer. We add that, about any point of the flow domain, there exists a neighborhood in which the complex variables $y_{c}$ and $c$ are in one-to-one correspondence. Without loss of generality, we will take $U^{\prime}(y)>0$ when $y \in[-1,1]$ for definiteness.

Rayleigh's equation has a singular point at the critical layer, but this is not the only possible singular point. First, there may be other points in the complex $y$ plane for which $U(y)=c$. Second, although no others exist when $U$ is continued into a neighborhood of $[-1,1]$, it is likely that additional singular points occur in $U$ when that function is further analytically continued into the complex plane. These singularities can then show up in Rayleigh's equation (though it is worth noting that because $U$ occurs in Rayleigh's equation only in the coefficient $U^{\prime \prime} /(U-c)$, for meromorphic $U$ all singular points in the finite complex plane are regular; this can easily be shown by Laurent expansion). However, here we will only need to consider the critical layer.

From the elementary theory of ordinary differential equations in the complex plane (see e.g. [11], Chap. X) it is known that there are two analytic solutions for $y$ in a neighborhood of any ordinary point. Moreover, in general, branch points occur in the solution at the locations of regular singular points. If we fix the two parameters, $k$ and $c$, then the locations of the singular points are fixed for a given equilibrium profile.

In a neighborhood of the critical point in the complex $y$-plane it is straightforward to obtain two Frobenius series solutions of the following form:

$$
\psi_{g}(y, c)=[U(y)-c] \varphi_{1}(y, c)
$$

and

$$
\psi_{b}(y, c)=[U(y)-c] \log |U(y)-c| \varphi_{2}(y, c)+\varphi_{3}(y, c),
$$

where $\varphi_{1}, \varphi_{2}$, and $\varphi_{3}$ are analytic for $y$ in this neighborhood and for $c$ in the finite complex $c$-plane. 
The Frobenius solutions can be used to construct neutral eigenmodes (with $c$ real). We will go about this construction shortly; however, Rayleigh's equation may also have complex eigenmode solutions, for which $c$ is complex, and we consider these first.

\subsection{Complex eigenmodes}

When $c$ is complex, there is no singular point in Rayleigh's equation for $y \in[-1,1]$, and we may then eliminate $\omega$ between (15) and (7) to obtain an integral equation for the streamfunction (eigenfunction) of the complex eigenmodes:

$$
\psi(y, c)=\int_{-1}^{1} \mathcal{G}\left(y, y^{\prime}\right) \frac{U^{\prime \prime}\left(y^{\prime}\right) \psi\left(y^{\prime}, c\right)}{U\left(y^{\prime}\right)-c} d y^{\prime} .
$$

This has the form of a Fredholm equation, and if there is a solution for $c=c_{D}$, that solution is known to be unique (e.g. [12]). Moreover, the conjugate, $c=c_{D}^{*}$, is also a solution. These complex conjugates comprise a pair of discrete eigenvalues, of which there can only be a finite number. In fact, at the end of this paper, we essentially give an upper bound on their number.

The complex pairs that satisfy (18) are equivalent to the homogeneous solutions of (10). Alternatively, the values $c=c_{D}$ and $c=c_{D}^{*}$ are the zeros of the Wronskian (14) of the Laplace transform theory.

According to the Rayleigh-Fjørtoft Theorem, instabilities occur only if the velocity profile contains a minimum in vorticity (since we assume $U^{\prime}>0$, the vorticity is everywhere negative). Thus, because $\operatorname{Im} c>0$ signifies instability, the theorem implies that the complex, discrete eigenmodes can only exist if the vorticity has a minimum.

\subsection{Neutral discrete eigenmodes}

The solution of Rayleigh's problem for the neutral eigenmodes is not so clear cut. In fact, if $c$ is real, then there can be no nontrivial, regular solutions for the streamfunction, with only a single exceptional type of mode. This follows from two facts. First, if $c<U(-1)=U_{1}$ or $c>U(1)=U_{2}$, then Rayleigh's equation with the assumed boundary conditions has only a trivial solution. Thus neutral solutions must have critical layers. Second, if $c$ lies within the flow domain, $\left[U_{1}, U_{2}\right]$, then it can be shown that $\psi_{g}$ cannot satisfy the boundary conditions (e.g. [13]). Therefore, all neutral eigenmodes must contain the "bad" solution $\psi_{b}(y, c)$, in which case $\psi(y, c)$ must have an undefined derivative. This failure to construct real analytic neutral eigenfunctions is connected to the presence of a continuous spectrum that occurs for wavespeeds lying inside the flow domain: $c \in[U(-1), U(1)]$ $[7]$.

The exceptional class of modes are those for which the critical layer lines up with an inflexion point of the equilibrium flow profile. In this case, $U^{\prime \prime}\left(y_{c}\right)=0$, and the singular point is removed from Rayleigh's equation. The Frobenius solution, $\psi_{b}(y, c)$, is then an analytic function. The exceptional modes also satisfy equation (18), but now $c=U\left(y_{c}\right)=U\left(y_{I}\right)$, where $y_{I}$ is the location of an inflection point. We call these eigensolutions, "inflection-point modes." These are discrete eigenmodes embedded in the continuous spectrum.

One important feature of the inflection-point modes is that they must be the limits of the complex pairs as $\operatorname{Im}(c) \rightarrow 0$. In fact, they are the basis of the Tollmien-Lin perturbation theory, which builds the nearby complex solutions from the inflection-point mode. This perturbation expansion is normally performed with an underlying assumption that the limit exists. Though this 
is largely a technicality, the assumption is strictly only valid when $U(y)$ is an analytic function on $[-1,1]$ (this is one of the results that comes directly out of the complex analysis described here).

\subsection{Singular eigensolutions}

Neutral eigenmodes cannot, therefore, be real analytic at the critical layer if $U^{\prime \prime}\left(y_{c}\right) \neq 0$. Consequently, because the derivative of the streamfunction is not defined for these modes, we can only impose continuity on such solutions to Rayleigh's equation. Thus $\psi(y, c)$ may have an arbitrary jump in its first derivative at $y=y_{c}$. Any family of such solutions can be taken to be a set of singular eigenfunctions of the continuous spectrum. However, there is one family that is especially useful, and we now construct these special singular eigenmodes.

The singular eigenfunctions are generalized function solutions to Rayleigh's equation (see [14] and [15]):

$$
\omega(y, c)=\mathcal{P} \frac{U^{\prime \prime} \psi}{U-c}+\lambda(c) \delta[U(y)-c],
$$

where $\mathcal{P}$ indicates the Cauchy Principal Value, $\delta(U-c)$ is Dirac's delta function, and $\lambda$ is yet to be determined. With the interpretation of the singularity in (19) by means of the Cauchy principal value, we may define the jump in the derivative of $\psi$ by $\lambda / U^{\prime}\left(y_{c}\right)$. This quantity is currently arbitrary and in particular can be taken to be any function of $c$. Our special singular eigenmodes arise from a judicious choice for $\lambda$.

If we integrate (19) across the channel, we obtain

$$
\Xi(c):=\int_{-1}^{1} \omega(y, c) d y=\mathcal{P} \int_{-1}^{1} \frac{U^{\prime \prime}(y) \psi(y, c)}{U(y)-c} d y+\frac{\lambda}{U_{c}^{\prime}},
$$

or

$$
\begin{aligned}
\frac{\lambda}{U_{c}^{\prime}}= & \int_{-1}^{1} \omega(y, c) d y-\mathcal{P} \int_{-1}^{1} \frac{U^{\prime \prime}(y) \psi(y, c)}{U(y)-c} d y \\
& =: \Xi(c)-\mathcal{P} \int_{-1}^{1} \frac{U^{\prime \prime}(y) \psi(y, c)}{U(y)-c} d y,
\end{aligned}
$$

where $U_{c}^{\prime}=U^{\prime}\left(y_{c}\right)$ and $\Xi(c)$ is a parameter that is, in general, a function of the eigenvalue. In fact, since $\omega$ is a generalized function, the amplitude of the eigenmode can only be fixed on multiplying by a suitable test function and integrating. In Eq. (21), the test function is simply unity, and so $\Xi$ can be regarded as the eigenfunction amplitude. Moreover, since this is a linear problem, we may choose the dependence of $\Xi$ as we wish; this then amounts to the selection of $\lambda$.

On using (7), (19), and (22), we find:

$$
\begin{aligned}
& \psi(y, c)=\frac{\lambda}{U_{c}^{\prime}} \mathcal{G}\left(y, y_{c}\right)+\mathcal{P} \int_{-1}^{1} \mathcal{G}\left(y, y^{\prime}\right) \frac{U^{\prime \prime}\left(y^{\prime}\right) \psi\left(y^{\prime}, c\right)}{U\left(y^{\prime}\right)-c} d y^{\prime} \\
= & \Xi(c) \mathcal{G}\left(y, y_{c}\right)+\int_{-1}^{1} \frac{\mathcal{G}\left(y, y^{\prime}\right)-\mathcal{G}\left(y, y_{c}\right)}{U\left(y^{\prime}\right)-c} U^{\prime \prime}\left(y^{\prime}\right) \psi\left(y^{\prime}, c\right) d y^{\prime} .
\end{aligned}
$$

Although (23) is at first sight a singular integral equation, (24) is a regular Fredholm equation of the second kind (e.g. [12]) that is straightforward to solve.

Provided the Fredholm equation has no homogeneous solution, (24) has a unique particular solution. If (24) does have a homogeneous solution, particular solutions are, in general, unbounded. 
The important point is that a family of well-behaved singular eigenmodes is needed to represent the continuous spectrum. If there are no homogeneous solutions, any choice for $\Xi(c)$ will suffice for this purpose. But if there are homogeneous solutions, a more specialized choice must be made. One possible selection is $\Xi(c)=\mathcal{D}(c)$, where $\mathcal{D}(c)$ is the Fredholm determinant. With this selection, the inhomogeneous term automatically vanishes if there is a homogeneous solution. This ensures that the solution to the Fredholm problem is always bounded. Hence, even if there are homogeneous solutions, we can always find a set of sensible singular eigenmodes. (Note that $\mathcal{D}\left(y_{c}\right)$ is determined by the kernel in (24), that is $\mathcal{G}\left(y, y^{\prime}\right)$ and $U(y)$, and is independent of the solution, $\psi(y, c)$, and so there is freedom for this selection for $\Xi(c)$.)

For Rayleigh's problem with the profiles we have considered, we have not found any homogeneous solutions to $(24)$. So $\Xi(c)=1$ is a convenient choice in any practical application. Then, from (19) and (22), we compute the singular eigenfunction and the jump in the streamfunction's derivative.

Finally, note that the amplitude of the solution at the critical layer, $\psi\left(y_{c}, c\right)$, cannot vanish, since the Frobenius solution (17) satisfies $\psi_{b}\left(y_{c}, c\right) \neq 0$. This is an important property of the singular eigenfunctions that will be used later.

\section{The dispersion relation}

In this section we construct an expression for the dispersion relation. The form of the dispersion relation is designed to facilitate the subsequent Nyquist analysis, and is one that is useful for relating discrete eigenmodes to continuum eigenmodes.

\subsection{Discontinuity and the dispersion relation}

We begin by considering an arbitrary point $y_{*}$ that is not coincident with the critical layer; i.e. $y_{*}$ is any ordinary point of the differential equation. For convenience we choose $y_{*} \in[-1,1]$ and we assume that $c$ is fixed in the upper half $c$-plane.

Now we construct two solutions, $\Psi_{<}(y, c)$ and $\Psi_{>}(y, c)$, that are defined for $y \in[-1,1]$ on the complex $c$-plane. The first, $\Psi_{<}(y, c)$, is defined by series expansion about the left boundary point $y=-1$ and satisfies the boundary condition $\Psi_{<}(-1, c)=0$. This is a one-parameter family of solutions, where the parameter can be taken to be a multiplicative constant; i.e. we can write $\Psi_{<}(y, c)=C_{<} \Phi_{<}(y, c)$, where $\Phi_{<}(y, c)$ is a parameter-independent solution of Rayleigh's equation that satisfies $\Phi_{<}(-1, c)=0$ and $\Phi_{<}^{\prime}(-1, c)=1$. Similarly, a second one-parameter family of solutions is constructed by series expansion about the point $y=1$. We denote this second solution by $\Psi_{>}(y, c)=C_{>} \Phi_{>}(y, c)$, where by construction $\Phi_{>}(1, c)=0$ and $\Phi_{>}^{\prime}(1, c)=1$. We emphasize that at fixed $y \in[-1,1]$, both $\Phi_{<}(y, c)$ and $\Phi_{>}(y, c)$ are analytic in $c$ for all $c \neq U(y)$.

The next step in our construction is to choose the constants $C_{<}$and $C_{>}$so that $\Psi_{<}$and $\Psi_{>}$are continuous at the point $y_{*} ;$ i.e. we set $\Psi_{<}\left(y_{*}, c\right)=\Psi_{>}\left(y_{*}, c\right)$. This requirement leaves a remaining constant that is an overall scaling factor; the two solutions can be represented as follows:

$$
\begin{aligned}
& \Psi_{<}\left(y, c, y_{*}\right)=C\left(c, y_{*}\right) \Phi_{>}\left(y_{*}, c\right) \Phi_{<}(y, c) \\
& \Psi_{>}\left(y, c, y_{*}\right)=C\left(c, y_{*}\right) \Phi_{<}\left(y_{*}, c\right) \Phi_{>}(y, c),
\end{aligned}
$$

where the sole remaining constant is $C\left(c, y_{*}\right)$, in which we have included dependence upon $c$ and 
$y_{*}$. Clearly, we are free to choose $C\left(c, y_{*}\right)$ arbitrarily. We give a prescription for defining $C\left(c, y_{*}\right)$ shortly.

No matter which (nonzero) value is chosen for $C\left(c, y_{*}\right)$, the derivatives of the functions $\Psi_{<}$ and $\Psi_{>}$will not, for general values of $c$, match at the point $y_{*}$. However, in the event that they do match, the value of $c$ is an eigenvalue and the functions $\Psi_{<}$and $\Psi_{>}$define an eigenfunction. In fact, when this is the case, $\Psi_{<}$and $\Psi_{>}$are analytic continuations of each other. This follows because $y_{*}$ is an ordinary point and both $\Psi_{<}$and $\Psi_{>}$are solutions of the Cauchy problem for Rayleigh's equation with identical specification of their values and derivatives at $y_{*}$. Moreover, when the derivatives of the solutions match, the point $y_{*}$ is immaterial and the dependence upon $y_{*}$ drops out of the incipient eigenfunction.

In light of the above, the jump in the derivatives of $\Psi_{<}$and $\Psi_{>}$at $y_{*}$ is equivalent to a dispersion relation: its vanishing determines $c=c(k)$. (Recall that the $k$ dependence has been suppressed in the expressions above.) Note that if $C\left(c, y_{*}\right)$ is chosen to be an analytic function of $c$ in the upper half plane, then $\Psi_{<}\left(y_{*}, c\right)$ and $\Psi_{>}\left(y_{*}, c\right)$ are also analytic functions of $c$ in the upper half plane, which follows from the assumption $U\left(y_{*}\right) \neq c$. Hence, the dispersion relation is an analytic function for $c$ in the upper half plane. Also, note that the dispersion relation does not depend upon the point $y_{*}$, since matching of the derivatives at any point will give an eigenfunction.

\subsection{Relationship between $\epsilon$ and $W$}

We now obtain a convenient expression for the dispersion relation.

In general, for $y$ within the flow domain, we write the streamfunction in the form,

$$
\Psi\left(y, c, y_{*}\right):=H\left(y-y_{*}\right) \Psi_{>}\left(y, c, y_{*}\right)+H\left(y_{*}-y\right) \Psi_{<}\left(y, c, y_{*}\right)
$$

and $H$ is the Heaviside function. The vorticity, by which we mean $\omega\left(y, c, y_{*}\right)=\Psi^{\prime \prime}-k^{2} \Psi$, then has a representation,

$$
\omega\left(y, c, y_{*}\right)=\Omega\left(y, c, y_{*}\right)+\tilde{\epsilon}\left(c, y_{*}\right) \delta\left(y-y_{*}\right) .
$$

where $\tilde{\epsilon}\left(c, y_{*}\right)$ measures the jump in the derivatives,

$$
\tilde{\epsilon}\left(c, y_{*}\right):=\Psi_{>}^{\prime}\left(y_{*}, c, y_{*}\right)-\Psi_{<}^{\prime}\left(y_{*}, c, y_{*}\right),
$$

and

$$
\Omega\left(y, c, y_{*}\right)=\left\{\begin{array}{l}
U^{\prime \prime}(y) \Psi_{<}\left(y, c, y_{*}\right) /[U(y)-c] \quad \text { if } y>y_{*} \\
U^{\prime \prime}(y) \Psi_{>}\left(y, c, y_{*}\right) /[U(y)-c] \quad \text { if } y<y_{*} .
\end{array}\right.
$$

By inserting (25) into (28) we see that

$$
\begin{aligned}
\tilde{\epsilon}\left(c, y_{*}\right) & =C\left(c, y_{*}\right)\left[\Phi_{>}\left(y_{*}, c\right) \Phi_{<}^{\prime}\left(y_{*}, c\right)-\Phi_{<}\left(y_{*}, c\right) \Phi_{>}^{\prime}\left(y_{*}, c\right)\right] \\
& =: C\left(c, y_{*}\right) W(c)
\end{aligned}
$$

where $W$, the Wronskian for Rayleigh's equation, is independent of $y_{*}$. Note that this quantity can be identified with the Wronskian of (13) by substituting $p=-i k c$ into the latter.

We next normalize the solution by integrating (27) over the flow domain and setting the result equal to the $c$-dependent parameter, $\tilde{\Xi}\left(c, y_{*}\right)$ :

$$
\int_{-1}^{+1} \omega\left(y, c, y_{*}\right) d y=\tilde{\Xi}\left(c, y_{*}\right),
$$


which yields

$$
\tilde{\Xi}\left(c, y_{*}\right)=\tilde{\epsilon}\left(c, y_{*}\right)+\int_{-1}^{y_{*}} \frac{U^{\prime \prime}(y) \Psi_{<}\left(y, c, y_{*}\right)}{U(y)-c} d y+\int_{y_{*}}^{1} \frac{U^{\prime \prime}(y) \Psi_{<}\left(y, c, y_{*}\right)}{U(y)-c} d y .
$$

By using Rayleigh's equation, we then find

$$
\tilde{\Xi}=-\Psi_{<}^{\prime}\left(-1, c, y_{*}\right)+\Psi_{>}^{\prime}\left(1, c, y_{*}\right)-k^{2} \int_{-1}^{y_{*}} \Psi_{<}\left(y, c, y_{*}\right) d y-k^{2} \int_{y_{*}}^{1} \Psi_{>}\left(y, c, y_{*}\right) d y .
$$

Finally, by inserting (25) into (33) we may express $C\left(c, y_{*}\right)$ in terms of $\tilde{\Xi}\left(c, y_{*}\right)$ (or vice versa):

$$
\begin{aligned}
C\left(c, y_{*}\right)=-\tilde{\Xi}\left(c, y_{*}\right)\left[k^{2} \int_{-1}^{y_{*}} \Phi_{>}\left(y_{*}, c\right) \Phi_{<}(y, c) d y\right. & +k^{2} \int_{y_{*}}^{1} \Phi_{<}\left(y_{*}, c\right) \Phi_{>}(y, c) d y \\
& \left.+\Phi_{>}\left(y_{*}, c\right)-\Phi_{<}\left(y_{*}, c\right)\right]^{-1}
\end{aligned}
$$

Because $\Phi_{<}$and $\Phi_{>}$are analytic in $c$ in the upper half plane for fixed $y$, the quantity in the denominator of the right-hand side of (34) is also analytic. Thus, if we were to select $C\left(c, y_{*}\right)$ to be analytic in the upper half $c$-plane, then $\tilde{\Xi}\left(c, y_{*}\right)$ would also be. However, the converse is not quite true: if $\tilde{\Xi}\left(c, y_{*}\right)$ is selected to be analytic in the upper half plane, then $C\left(c, y_{*}\right)$ is also analytic except, perhaps, for poles at the zeros of the denominator. We will interpret these zeros shortly.

\subsection{Rayleigh Green function and singular eigenfunctions}

The next piece of the puzzle is to relate the dispersion relation to the singular eigenfunctions.

We may rewrite (27) in the form,

$$
\Psi^{\prime \prime}-k^{2} \Psi-\frac{U^{\prime \prime} \Psi}{U-c}=\tilde{\epsilon} \delta\left(y-y_{*}\right)
$$

Thus, $\Psi$ is related to the Green function for Rayleigh's equation. This equation is a bit subtle, since $\tilde{\epsilon}$ (according to (32)) is in fact a property of the solution and so the equation is self-referential. However, if $y_{*} \neq y_{c}$ and $\tilde{\epsilon} \neq 0$, then $\Psi / \tilde{\epsilon}$ satisfies the normal equation for the Green function.

We may turn equation (35) into one of integral form on using the Green function of the Laplacian:

$$
\Psi\left(y, c, y_{*}\right)=\mathcal{G}\left(y, y_{*}\right) \tilde{\epsilon}\left(c, y_{*}\right)+\int_{-1}^{1} \mathcal{G}\left(y, y^{\prime}\right) \frac{U^{\prime \prime}(y) \Psi\left(y^{\prime}, c, y_{*}\right)}{U\left(y^{\prime}\right)-c} d y^{\prime}
$$

Moreover, on using the relation (32), this can be written as

$$
\Psi\left(y, c, y_{*}\right)=\tilde{\Xi}\left(c, y_{*}\right) \mathcal{G}\left(y, y_{*}\right)+\int_{-1}^{1} \Psi\left(y^{\prime}, c, y_{*}\right) \mathcal{K}\left(y, y^{\prime}, y_{*}, c\right) d y^{\prime}
$$

where

$$
\mathcal{K}\left(y, y^{\prime}, y_{*}, c\right):=U^{\prime \prime}\left(y^{\prime}\right) \frac{\left[\mathcal{G}\left(y, y^{\prime}\right)-\mathcal{G}\left(y, y_{*}\right)\right]}{U\left(y^{\prime}\right)-c} .
$$

Hence the Green function can be constructed by solving another Fredholm integral equation. 
In fact, the two Fredholm problems are closely related: if we take the limit $c \rightarrow c_{r}+i 0 \equiv$ $U\left(y_{c}\right)+i 0$ with $y_{*}=y_{c}$, then we recover the Fredholm equation for the singular eigenfunctions from (37)-(38) with $\Psi\left(y, c_{r}+i 0, y_{c}\right)=\psi\left(y, c_{r}\right)$ and $\tilde{\Xi}\left(c, y_{c}\right) \equiv \Xi(c)$.

Similarly, we may recover the singular eigenfunction (19) from the Green function equation (27) in the same limit: we let $c \rightarrow c_{r}+i 0 \equiv U\left(y_{c}\right)+i 0$ in (32), giving

$$
\tilde{\epsilon}\left(c_{r}+i 0, y_{c}\right)=\tilde{\Xi}\left(c_{r}+i 0, y_{c}\right)-\int_{-1}^{1} \frac{U^{\prime \prime}(y) \Psi\left(y, c_{r}+i 0, y_{c}\right)}{U(y)-c_{r}-i 0} d y
$$

However, since $\Psi\left(y, c, y_{*}\right)$ is analytic in the upper half plane, there exists a generalized form of the Plemelj relation (e.g. [16]), which leads to

$$
\begin{gathered}
\tilde{\epsilon}\left(c_{r}+i 0, y_{c}\right)=\Xi\left(c_{r}\right)-\mathcal{P} \int_{-1}^{1} \frac{U^{\prime \prime}(y) \psi\left(y, c_{r}\right)}{U(y)-c_{r}} d y-i \pi \frac{U^{\prime \prime}\left(y_{c}\right) \psi\left(y_{c}, c_{r}\right)}{U^{\prime}\left(y_{c}\right)} \\
=: \tilde{\epsilon}_{r}\left(c_{r}, y_{c}\right)+i \tilde{\epsilon}_{i}\left(c_{r}, y_{c}\right),
\end{gathered}
$$

on using the association of the Fredholm equations to replace $\Psi\left(y, c_{r}+i 0, y_{c}\right)$ with $\psi\left(y, c_{r}\right)$. The Plemelj relation also implies that

$$
\omega\left(y, c_{r}+i 0, y_{c}\right)=\mathcal{P} \frac{U^{\prime \prime} \psi\left(y, c_{r}\right)}{U-c_{r}}+\tilde{\epsilon}_{r}\left(c_{r}, y_{c}\right) \delta\left(y-y_{c}\right) .
$$

Finally, we identify $\omega\left(y, c_{r}+i 0, y_{c}\right)$ with $\omega\left(y, c_{r}\right), \tilde{\epsilon}\left(c_{r}, y_{c}\right)$ with $\epsilon\left(c_{r}\right)$, and $\tilde{\epsilon}_{r}\left(c_{r}, y_{c}\right)$ with $\lambda(c) / U_{c}^{\prime}$. With these associations understood, we will drop the tildes in the next section and the extra argument in $y_{c}$.

Note that we could take a completely different approach to the problem beginning from the Green function. First we select $\tilde{\Xi}\left(c, y_{*}\right)$ to be a function that is suitably analytic in $c$. Then, the solution of the integral equation (37) can be shown to have the various analyticity properties we have found for $\Psi\left(y, c, y_{*}\right)$ by using Fredholm theory (the kernel is an analytic function of the parameter $c$ in the upper half plane). From there, we build the quantity $\tilde{\epsilon}\left(c, y_{*}\right)$, which we know has zeros if $c$ is an eigenvalue. Moreover, equation (34) determines a function $C\left(c, y_{*}\right)$ by which we may relate the solution of the integral problem to the solutions $\Phi_{<}$and $\Phi_{>}$used above.

The only fly in the ointment is the possibility of a homogeneous solution to the Fredholm problem for some value of $c$, in which case the particular solution we seek is unbounded. From Fredholm theory we know that the solution $\Psi$ is not analytic at this value of $c$, and, in fact, has a pole.

However, this nonanalyticity is evidently an artifact of solving the Fredholm problem, since the solutions $\Phi_{<}$and $\Phi_{>}$are analytic in $c$. Moreover, since they are related to the solution $\Psi$ of (37) simply by the factor $C\left(c, y_{*}\right)$ as in equation $(25)$, it is clear that the nonanalyticity in $\Psi\left(y, c, y_{*}\right)$ is equivalent to a pole in $C\left(c, y_{*}\right)$. But since we may choose $\tilde{\Xi}\left(c, y_{*}\right)$ to be analytic, the pole must arise from a zero in the denominator of (34). This is the advertized interpretation of the poles in $C\left(c, y_{*}\right)$; they correspond to the presence of a homogeneous solution to the Fredholm problem (37).

At this stage, two remarks are in order. We have already noted that there are no homogeneous solutions for $c$ in the flow domain in the context of the singular eigenfunction equation. Hence, $C\left(c, y_{*}\right)$ must be real and contain no poles for these values of $c$. Secondly, since this nonanalyticity is $\Psi\left(y, c, y_{*}\right)$ is purely an artifact of solving the Fredholm problem (37), we can take a slightly different tack and make a judicous choice for $\tilde{\Xi}\left(c, y_{*}\right)$ that avoids the problem. Again, this is just a 
choice like $\tilde{\Xi}=\mathcal{D}\left(c, y_{*}\right)$, where $\mathcal{D}\left(c, y_{*}\right)$ is the Fredholm determinent of the kernel in (37). Once we make this choice, we may derive a solution of the integral equation that is analytic in $c$ in the upper half plane, and use it to build the dispersion relation through $\tilde{\epsilon}\left(c, y_{*}\right)$. However, we will explicitly follow the route outlined earlier in this section rather than this Green-function based avenue.

\section{$5 \quad$ Nyquist method}

In the previous section we discussed the functions $\epsilon(c)$ and $W(c)$, and their relationship. If their exists a discrete value of the phase velocity, $c=c_{D}$, such that $W\left(c_{D}\right)=0$, then we have an exponentially growing eigenmode with growth rate, $\operatorname{Im}\left(k c_{D}\right)$. By construction we know that $W(c)$ is analytic for $c$ in the upper half plane, and that it also has a branch cut along the real axis between $U_{1}:=U(-1)$ and $U_{2}:=U(+1)$. This branch cut arises from that of the natural logarithm of the Frobenius solution and the matching procedure (cf. (75) of the Appendix.) Because of these properties we begin with $W$ in our analysis below, but in the end we express the final result in terms of $\epsilon$, a quantity that is by design reminiscent of the dispersion relation of Vlasov theory.

The Nyquist method relies upon the argument principle of complex analysis. In the present context this principle implies that the integral,

$$
\frac{1}{2 \pi i} \int_{\mathcal{C}} \frac{W^{\prime}(c)}{W(c)} d c
$$

counts the number of zeros of $W$ in the region enclosed by a closed contour $\mathcal{C}$ in the $c-$ plane. We choose $\mathcal{C}$ to run along the $c_{r}$ axis, with $c_{i}$ fixed to an arbitrarily small positive value, and then the contour is closed by a large semicircular portion as shown in Fig. 2. As the radius of the semicircle goes to infinity, (43) gives the number of exponentially growing eigenvalues. Equivalently, (43) is the number of times the path determined by the function $W(c)$ encircles the origin of the $\left(W_{r}, W_{i}\right)$ plane as $c$ completes a circuit of $\mathcal{C}$.

It is efficacious to decompose the integral (43) into the following two pieces:

$$
\frac{1}{2 \pi i} \int_{\mathcal{C}} \frac{W^{\prime}(c)}{W(c)} d c=\frac{1}{2 \pi i} \int_{U_{1}+i 0}^{U_{2}+i 0} \frac{W^{\prime}(c)}{W(c)} d c+\frac{1}{2 \pi i} \int_{\mathcal{C}^{\prime}} \frac{W^{\prime}(c)}{W(c)} d c .
$$

The first integral is all important, while the second is relatively minor. To understand this recall Howard's semicircle theorem [8], which states that the zeros of $W$ must lie within a disk of radius, $\left(U_{1}+U_{2}\right) / 2$, centered at the point $\left(U_{2}-U_{1}\right) / 2$ (as illustrated in Fig. 2). Hence, if $\mathcal{C}$ encloses the semicircle, it contains all of the unstable eigenvales. In fact, wherever $W$ is analytic, we may deform the contour $\mathcal{C}^{\prime}$. Since $W$ is analytic in the upper half plane we may deform $\mathcal{C}^{\prime}$ into any contour there that connects $W\left(U_{1}+i 0\right)$ to $W\left(U_{2}+i 0\right)$ The important point is that since $\mathcal{C}^{\prime}$ is deformable to any other contour lying outside Howard's semicircle, the path defined by $W(c)$ as $c$ varies along $\mathcal{C}^{\prime}$ cannot lead to a new enclosure of the origin because this would mean a zero of $W$ outside the semicircle. In other words, the count of unstable eigenvales must be independent of the integration around $\mathcal{C}^{\prime}$; the only importance of the integral over $\mathcal{C}^{\prime}$ is to complete a closed path in the $\left(W_{r}, W_{i}\right)$ plane without encircling the origin. In the Appendix we demonstrate this explicitly by extending the contour $\mathcal{C}^{\prime}$ to infinity and then by analyzing the image of its various pieces in the $W$-plane. The upshot is that we may ignore the $\mathcal{C}^{\prime}$ part of the contour: the change in the argument of $\mathrm{W}$ along the flow domain is equal to the number of times the function $W(c)$ encircles the origin, which is equal to the number of unstable eigenvalues. 


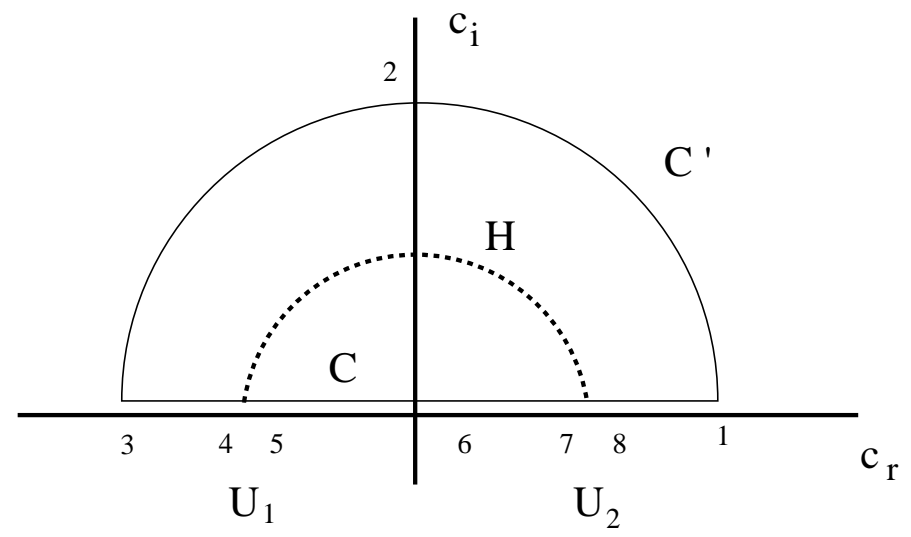

Figure 2: The contours $\mathcal{C}, \mathcal{C}^{\prime}$, and $H$ in the $c$-plane. The closed contour $\mathcal{C}$ runs the entire circuit from $1 \rightarrow 2 \rightarrow \ldots \rightarrow 8 \rightarrow 1$, with the portion from $3 \rightarrow 1$ lifted infinitesimally above the real axis. The contour $\mathcal{C}^{\prime}$ is $\mathcal{C}$ with the piece along the flow domain, $5 \rightarrow 7$, removed. The contour $H$ (dashed) is Howard's semicircle, within which the unstable eigenvalues must lie.

In Section 4 we showed that $\epsilon$ and $W$ are related by $\epsilon(c)=C(c) W(c)$. But as $c$ traverses the flow domain:

$$
\int_{U_{1}+i 0}^{U_{2}+i 0} \frac{\epsilon^{\prime}}{\epsilon} d c=\int_{U_{1}+i 0}^{U_{2}+i 0} \frac{W^{\prime}}{W} d c+\int_{U_{1}+i 0}^{U_{2}+i 0} \frac{C^{\prime}}{C} d c
$$

Upon introducing

$$
\epsilon=|\epsilon| e^{i \arg [\epsilon]} \quad \text { and } \quad W=|W| e^{i \arg [W]},
$$

(45) becomes

$$
\left.(\ln |\epsilon|+i \arg [\epsilon])\right|_{U_{1}+i 0} ^{U_{2}+i 0}=\left.(\ln |W|+\ln C+i \arg [W])\right|_{U_{1}+i 0} ^{U_{2}+i 0} .
$$

Now, as remarked at the end of Section $4, C(c)$ is real and finite along this part of the contour. Hence, equating imaginary parts gives $\Delta \arg [\epsilon]=\Delta \arg [W]$.

Therefore, the number of unstable eigenvalues is determined by the change in the argument of the following quantity:

$$
\begin{gathered}
\epsilon\left(c_{r}+i 0\right)=1-\mathcal{P} \int_{-1}^{1} \frac{U^{\prime \prime}(y) \psi(y, c)}{U(y)-U\left(y_{c}\right)} d y-i \pi \frac{U^{\prime \prime}\left(y_{c}\right) \psi\left(y_{c}, c\right)}{U^{\prime}\left(y_{c}\right)} \\
=\epsilon_{r}\left(c_{r}\right)+i \epsilon_{i}\left(c_{r}\right),
\end{gathered}
$$

where $c_{r}=U\left(y_{c}\right)$ (and we have explicitly made the choice $\Xi(c)=1$ ), which is the "Nyquist function" of Eq. (2). As $c_{r}$ varies from $U_{1}$ to $U_{2}$, or equivalently, $y_{c}$ from -1 to $1, \epsilon\left(c_{r}\right)$ executes a closed path in the $\left(\epsilon_{r}, \epsilon_{i}\right)$ plane. This path begins and ends at $(1,0)$ since $\psi( \pm 1, c)=0$. In between, the path circulates around and the following considerations help us determine whether or not the origin is encircled.

The path can only cross the $\epsilon_{r}$-axis at the points for which $\epsilon_{i}=0$, or $\psi\left(y_{c}, c_{r}\right) U^{\prime \prime}\left(y_{c}\right)=0$. However, as noted at the end of Section $3, \psi\left(y_{c}, c_{r}\right)$ cannot vanish. Hence the crossing points 
are just the inflection points of the velocity profile. We denote these by $y_{I}$, with $U^{\prime \prime}\left(y_{I}\right)=0$ and $c=c_{I}=U\left(y_{I}\right)$. Thus, the path emerges from the point $(1,0)$ on the $\left(\epsilon_{r}, \epsilon_{i}\right)$ plane, circulates around crossing the $\epsilon_{r}$-axis as many times as there are inflection points, and then terminates at $(1,0)$. If the origin is encircled, we have an exponentially growing instability. Hence, by calculating the singular eigenfunctions from the Fredholm problem (24) and then constructing $\epsilon\left(c_{r}+i 0\right)$, we can determine if there is an unstable eigenvalue.

In fact, the path can only enclose the origin if there is at least one crossing point to the left of the origin. Such a crossing point is guaranteed if

$$
\int_{-1}^{1} \frac{U^{\prime \prime}(y) \psi\left(y, c_{I}\right)}{U(y)-U\left(y_{I}\right)} d y>1
$$

for at least one of the inflection points.

Inequality (50) is actually a sufficient condition for instability. We see this as follows: if there are an odd number of crossings to the left of the origin, then (50) certainly guarantees an enclosure of the origin. But if there are an even number, then one can envision paths that cross to the left of the origin, but do not enclose it. In this case, (50) may still hold, but the locus fails to encircle the origin. However, the condition still predicts instability because we may yet vary $k$, and the path must change continuously as we change $k$. From the Fredholm problem, one can observe that $\psi \sim 1 / k$ for large $k$. This means that $\psi \rightarrow 0$ as $k \rightarrow \infty$. Thus for large enough $k$, the path of $\epsilon$ shrinks about the point $(1,0)$. Since varying $k$ also cannot destroy crossing points, it must be the case that there is a range of values of $k$ for which two of the crossing points must straddle the origin, and the path encircles it. (An example exhibiting this feature is given in Section 7.3.)

Thus (50) guarantees an enclosure of the origin for some value of $k$, and for any number of crossings to the left of the origin. In other words, we deduce the following necessary and sufficient condition for instability:

Rayleigh's equation possess an unstable eigenmode if and only if there exists a wavenumber, $k$, and at least one inflection point, $y_{I}$, such that Eq. (50) holds.

Note that, by "crossing point" we mean strictly only those inflection points for which $U^{\prime \prime \prime} \neq 0$. This implies that intersection of the path with the axis leads to a traversal of the axis from one half plane to the other. Inflection points for which $U^{\prime \prime \prime}=0$ can lead to the path touching but not crossing the axis, and these intersections will not signify a nearby enclosure of the origin. However, an arbitrarily small deformation of $U(y)$ can in this situation lead to the locus encircling the origin. Thus profiles with $U^{\prime \prime \prime}=0$ correspond to marginally stable states (see Section 7 ).

Unfortunately, the sufficient condition implied by (50) has a more complicated dependence on $k$ than in the related Vlasov problem (the $k$-dependence in the Vlasov problem is contained purely in a multiplicative factor). The dependence here comes from the dependence of $\psi\left(y, c_{I}\right)$ on $k$, which is not obvious. Nevertheless, if $k^{2}>k_{m}^{2}:=-\operatorname{Min}\left[U^{\prime \prime} /\left(U-c_{I}\right)\right]$ for all of the inflexion points $y_{I}$, then we can apply a result of Howard [17] which indicates that there can be no instability. Hence for $k>k_{m}$, the crossing point must lie to the right of the origin, so we can at least bound the range of interesting wavenumbers from above.

Finally, the condition (50) refers only to exponentially growing eigenmodes and, therefore, does not encompass all the possibilities for instability. It fails to account for a possible algebraic instability of the continuous spectrum. However, in the present context, we can safely ignore such a possibility since we know that the continuum is stable for a monotonic velocity profile [9]. Therefore, 
algebraic instability is ruled out. Hence (50) is the encompassing condition for instability. This may not be true if the profile is nonmonotonic (see [18]).

\section{Special cases}

In the previous section we described our necessary and sufficient condition for instability; here we illustrate our criterion for two special examples: general profiles with $k=0$, and an asymptotic result.

\subsection{General $k=0$ eigenmodes}

For an arbitrary profile with $k=0$, Rayleigh's equation simplifies and the eigenmodes can be found by directly integrating. It is straightforward to construct the Wronskian,

$$
W(c)=\left(U_{2}-c\right)\left(c-U_{1}\right) \int_{-1}^{1} \frac{d y}{(U-c)^{2}} .
$$

We could analyze this dispersion relation independently of the machinery developed in the previous sections. However, we consider the $k=0$ problem by way of illustration, and so we apply the general methodology.

The Fredholm problem (or the procedure of Section 4) for the streamfunctions of the singular eigenfunctions has in this case the analytical solution:

$$
\psi(y, c)=\left\{\begin{array}{cc}
-\left(U_{2}-c\right)\left(c-U_{1}\right)\left(U_{2}-U_{1}\right)^{-1} U_{c}^{\prime}(U-c) \int_{-1}^{y} d y^{\prime} /\left[U\left(y^{\prime}\right)-c\right]^{2} & \text { for } y<y_{c} \\
\left(U_{2}-c\right)\left(c-U_{1}\right)\left(U_{2}-U_{1}\right)^{-1} U_{c}^{\prime}(U-c) \int_{y}^{1} d y^{\prime} /\left[U\left(y^{\prime}\right)-c\right]^{2} & \text { for } y>y_{c} .
\end{array}\right.
$$

Consequently, the Nyquist function can be written in the form,

$$
\epsilon\left(c_{r}+i 0\right)=-\frac{\left(U_{2}-c_{r}\right)\left(c_{r}-U_{1}\right)}{U_{2}-U_{1}} U_{c}^{\prime} \int_{-1}^{1} \frac{d y}{\left[U(y)-c_{r}-i 0\right]^{2}},
$$

or

$$
\epsilon_{r}\left(c_{r}\right)=\frac{\left(U_{2}-c_{r}\right)\left(c_{r}-U_{1}\right)}{U_{2}-U_{1}} U_{c}^{\prime}\left\{\frac{1}{\left(U_{2}-c_{r}\right) U_{2}^{\prime}}+\frac{1}{\left(c_{r}-U_{1}\right) U_{1}^{\prime}}+\mathcal{P} \int_{-1}^{1} \frac{U^{\prime \prime}(y) d y}{\left[U(y)-c_{r}\right] U^{\prime}(y)^{2}}\right\}
$$

and

$$
\epsilon_{i}\left(c_{r}\right)=-\pi \frac{\left(U_{2}-c_{r}\right)\left(c_{r}-U_{1}\right) U^{\prime \prime}\left(y_{c}\right)}{\left(U_{2}-U_{1}\right) U^{\prime}\left(y_{c}\right)^{3}}
$$

where $U_{1}^{\prime}=U^{\prime}(-1)$ and $U_{2}^{\prime}=U^{\prime}(1)$.

Note that, for the $k=0$ problem, $C(c)=-U_{c}^{\prime} /\left(U_{2}-U_{1}\right)$ for $c$ in the flow domain. Thus $C(c)$ is indeed real and finite, as we remarked earlier.

In the Nyquist construction, the important piece of the contour $\mathcal{C}$ leaves the point $(1,0)$, executes some path that is dependent on the details of $U(y)$, and finally returns to $(1,0)$. That is, the path is closed, as we remarked earlier. The Nyquist function of (53) is related to that obtained in [5], where this special case of $k=0$ was considered in a more specific fashion. Note, however, that the Nyquist function of [5] differs from (53) by a factor in front of the integral that leads to $\epsilon(c)$ vanishing at $c=U_{1}$ and $U_{2}$. If this were not so, our Nyquist plots would not be closed loops and one would be forced to consider the neglected piece of the contour, $C^{\prime}$, in detail. 


\subsection{An asymptotic result}

Our second example is only an approximate result; it concerns velocity profiles of the form, $U(y)=$ $y+\varepsilon^{2} \mathcal{U}(y / \varepsilon)$, where $\varepsilon \ll 1$. This kind of velocity profile represents a linear background profile with a superposed, sharply varying, "defect." To leading order in $\varepsilon$, the corresponding streamfunction is given by

$$
\psi(y, c)=\mathcal{G}\left(y, y_{c}\right)+O(\varepsilon)
$$

and the Nyquist function has the simple form

$$
\epsilon(c)=1-\frac{\tanh k}{2 k} \int_{-\infty}^{\infty} \frac{\mathcal{U}^{\prime \prime}(\eta) d \eta}{\eta-c}+O(\varepsilon),
$$

or

$$
\epsilon_{r}\left(c_{r}\right)=1-\frac{\tanh k}{2 k} \mathcal{P} \int_{-\infty}^{\infty} \frac{\mathcal{U}^{\prime \prime}(\eta) d \eta}{\eta-c_{r}}+O(\varepsilon)
$$

and

$$
\epsilon_{i}\left(c_{r}\right)=-\frac{\tanh k}{2 k} \mathcal{U}^{\prime \prime}\left(y_{c}\right)+O(\varepsilon) .
$$

This asymptotic result is given in [19], following [20]. It is closely related to the corresponding Vlasov solution.

Note that in the two examples, $U(y)$ need not be analytic on $[-1,1]$; in fact, the existence of two derivatives is sufficient.

\section{Numerical solutions for sample profiles}

We now construct some Nyquist plots numerically for a trio of sample profiles that illustrate different features of the inviscid stability problem.

\subsection{Single inflection point}

The profile,

$$
U(y)=\tanh \beta y,
$$

is an example of an equilibrium with a single inflection point. Nyquist plots for various values of $k$ and $\beta=2$ are drawn in Fig. 1(a). For wavenumbers $k<k_{c}$ with $k_{c} \simeq 1.832$, there is evidentally an unstable eigenvalue, since the Nyquist plots enclose the origin over this range of $k$. Plots for $k=0$ and different values of $\beta$ are shown in Fig. 1(b).

In Fig. 1(a), the Nyquist plot passes through the origin without encircling it for the critical value $k=k_{c}$. This wavenumber is the demarcation between stability and instability; that is, it is the stability boundary, $k_{c}=k_{c}(\beta)$. This stability boundary is displayed in Fig 3(a). As $\beta$ decreases, the critical wavenumber decreases until it vanishes at a special value of $\beta=\beta_{m} \simeq 1.2$. For the profile with this critical parameter value, the $k=0$ Nyquist plot passes through the origin and none encircle it. In other words, such a profile is a marginally stable state.

This feature of the profile is an example of a general result: instability always sets in first at $k=0$ for profiles with a single inflection point (e.g. [8]). Hence the instability condition is given by $\epsilon_{r}<0$ with $\epsilon_{r}$ given by Eq. (54). This is equivalent to the result of [5]. 
(a)

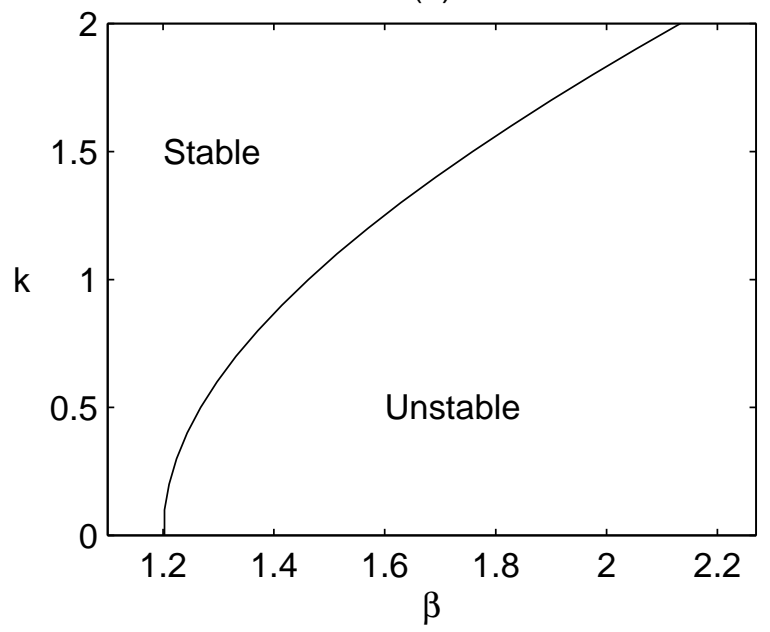

(c)

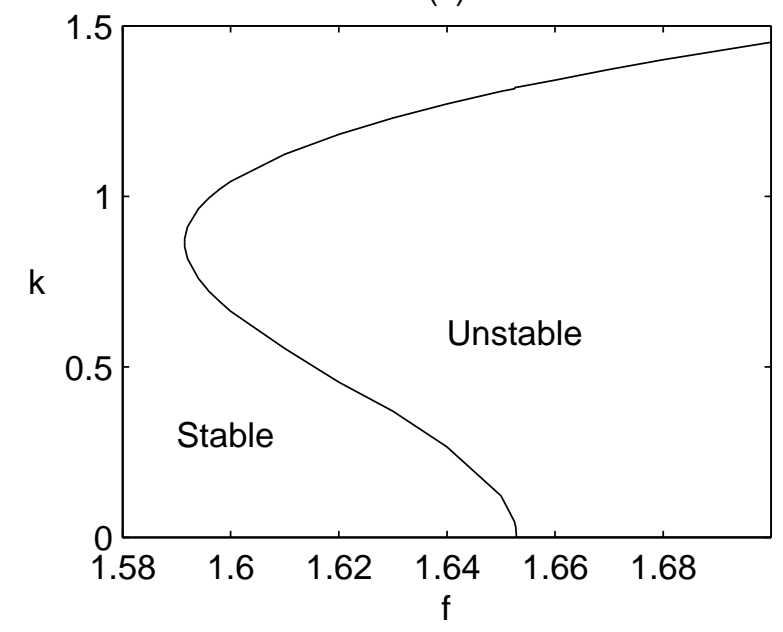

(b)

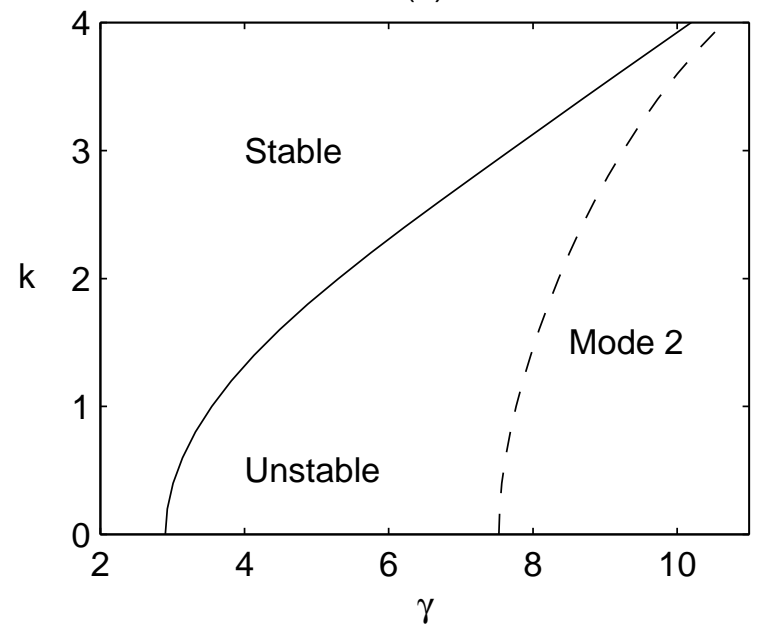

Figure 3: Stability boundaries for the three equilibrium profiles of Sec. 7. Figures (a) and (b) depict the nucleation of instability through $k=0$, while (c) depicts the nucleation of instability through $k \neq 0$. In (b), the stability boundary of the second and third unstable modes is also shown. 
In some applications, however, one may be interested in flows that are periodic in $x$ (such as in annular or spherical geometry, or in numerical simulations). In these cases, there is a minimum wavenumber, and $k=0$ is neither accessible nor relevant. Hence the $k=0$ theory is not applicable even for a single inflexion point. In this circumstance one must deal with the general Nyquist function and sufficient stability condition described in Section 5.

\subsection{Multiple inflection points}

The profile,

$$
U(y)=y+\frac{1}{2 \gamma} \sin \gamma y
$$

is an example in which there are multiple inflection points. In this case, the number of inflection points varies with $\gamma$. For $\gamma=5,7.5$, and 10, there are 3, 5, and 7 inflecton points, respectively. As $\gamma$ increases, the new inflexion points appear in pairs through the boundaries, $y= \pm 1$. The Nyquist plots at the three parameter values and $k=1$ are shown in Fig. 4(a)-(c). For these plots there are multiple encirclings of the origin, signifying multiple instabilities. For example, when $k=1$ and $\gamma=10$, there are three loops around the origin (see Fig. 4(c)). As $\gamma$ increases and inflexion points appear at the boundaries, the Nyquist curve acquires more loops that appear out of the asymptote $(1,0)$.

At $\gamma=10$, there are therefore three unstable eigenvalues with $k=1$, which is the most this profile can support (four of the inflection points lead to crossing of the $\epsilon_{r}$-axis, but these lie to the right of the asymptote $(1,0))$. Note that the antisymmetry of the profile means that the path of $\epsilon\left(c_{r}+i 0\right)$ is symmetric under reflection about the $\epsilon_{r}$-axis.

If we vary $k$, and calculate $\epsilon_{r}\left(c_{I}\right)$, where $c_{I}$ with $I=1,2,3$ denotes the three inflection points that lead to crossings to the left of the asymptote $(1,0)$, then we obtain the picture shown in Fig. 4(d). This shows that the $\epsilon_{r}\left(c_{I}\right)$ increase monotonically with $k$. This suggests that if we were to vary $\gamma$, then all three unstable eigenvalues would appear first at $k=0$, which is indeed true as can be seen from Fig. 3(b). Thus, the marginal state for this profile is again given by the $k=0$ theory.

\subsection{Finite wavenumber instabilities}

The third profile,

$$
U(y)=y+5 y^{3}+f \tanh 4(y-1 / 2),
$$

contains one or three inflection points, depending on the value of the parameter $f$. More specifically, as we increase $f$ through about 1.59 , we create two inflection points near the point $y=0.6$ (see Fig. 5). The critical profile for which the two inflection points emerge contains a point with $U^{\prime \prime}=U^{\prime \prime \prime}=0$. This leads to a Nyquist plot that contains a nontransversal intersection of the path with the $\epsilon_{r}$-axis; that is, the plot touches the axis but does not cross it. For larger values of $f$, this degenerate point splits into the two inflection points and the plot crosses the $\epsilon_{r}$-axis twice. Nyquist plots beyond this bifurcation are shown in Fig. 6(b).

This kind of a change to the profile leads to a situation in which there can be two crossings of the $\epsilon_{r}$-axis to the left of the origin, but in neighborhood of $k=0$ there are no unstable eigenvalues. Yet, as we remarked in Section 5, increasing $k$ leads to a deformation of the path such that the origin is eventually encircled for some range of $k$ (see Fig. 6(a) and (b)). In Fig. 7, we show the 
(a)

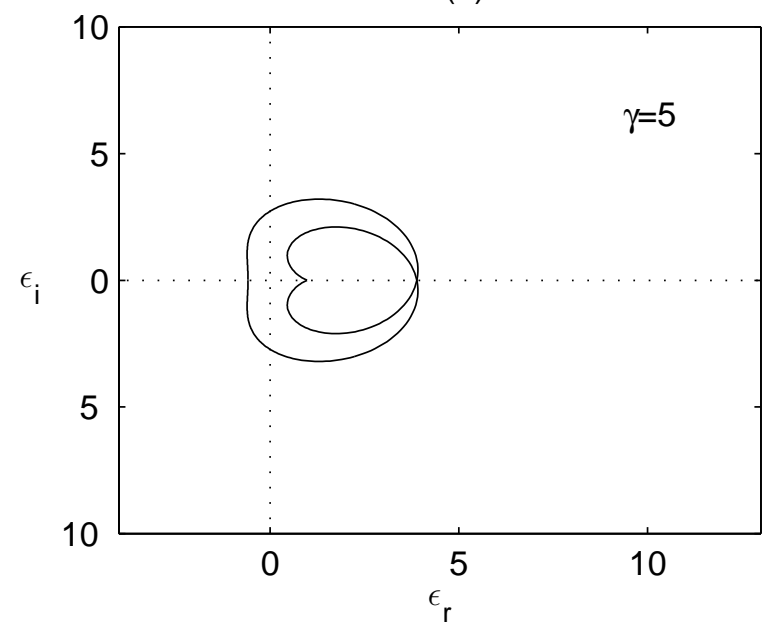

(c)

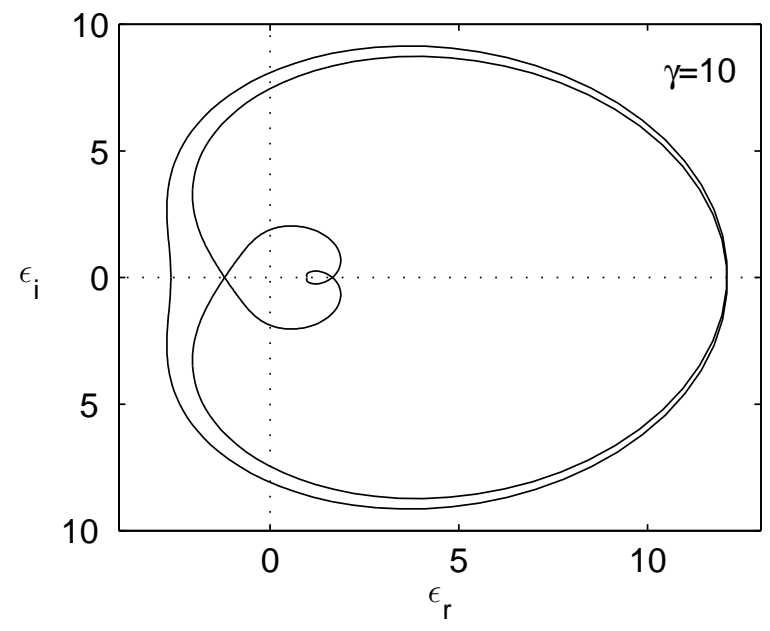

(b)

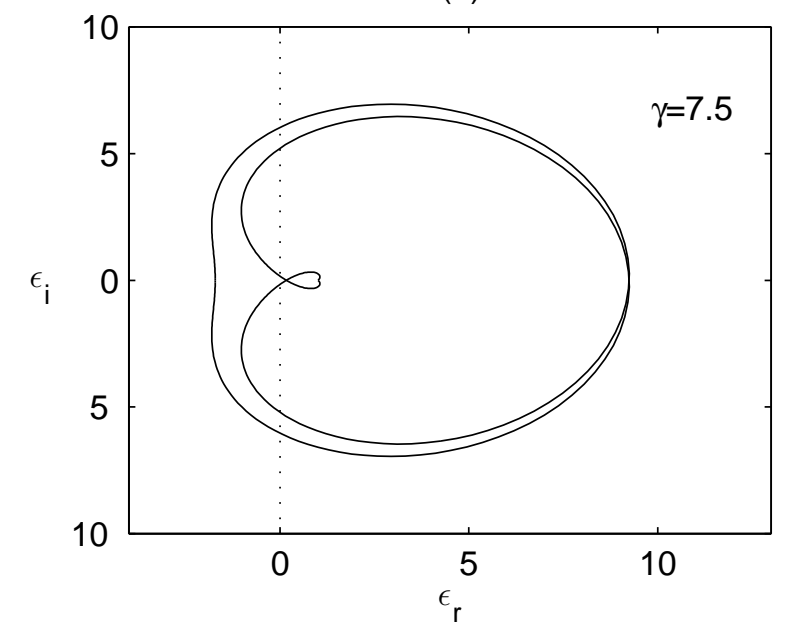

(d)

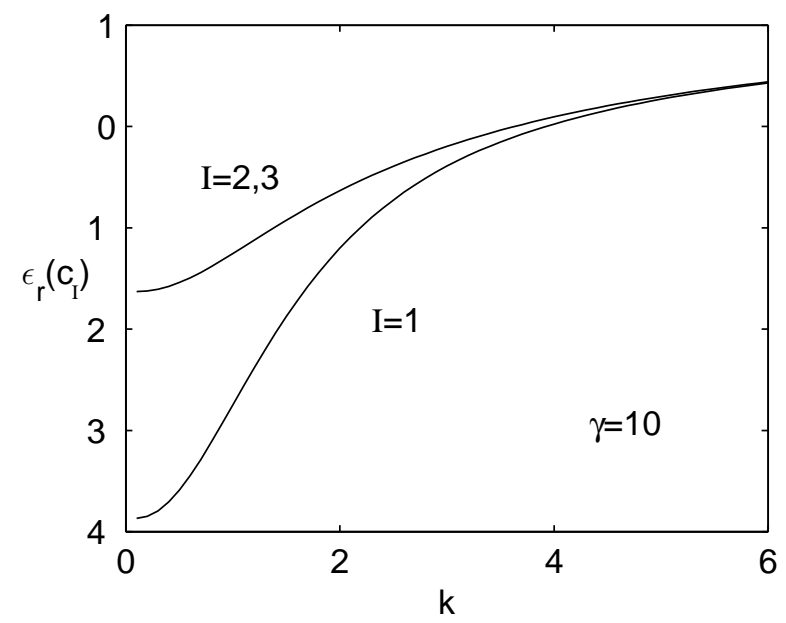

Figure 4: Nyquist plots for the multiple inflection point profile, $U(y)=y+(\sin \gamma y) / 2 \gamma$, with $k=1$. (a) The case $\gamma=5$ has one encircling of the origin and thus one unstable eigenvalue. (b) In the case with $\gamma=7.5$ two more loops have appeared and the curve almost encircles the origin two more times. That is, it is close to the threshold of the instability of two further modes. (c) The case $\gamma=10$ has three encirclings of the origin and three unstable eigenvalues. (d) Depiction of the monotonic dependence of the crossing values to the left of the asymptote $(1,0)$ as a function of $k$. 


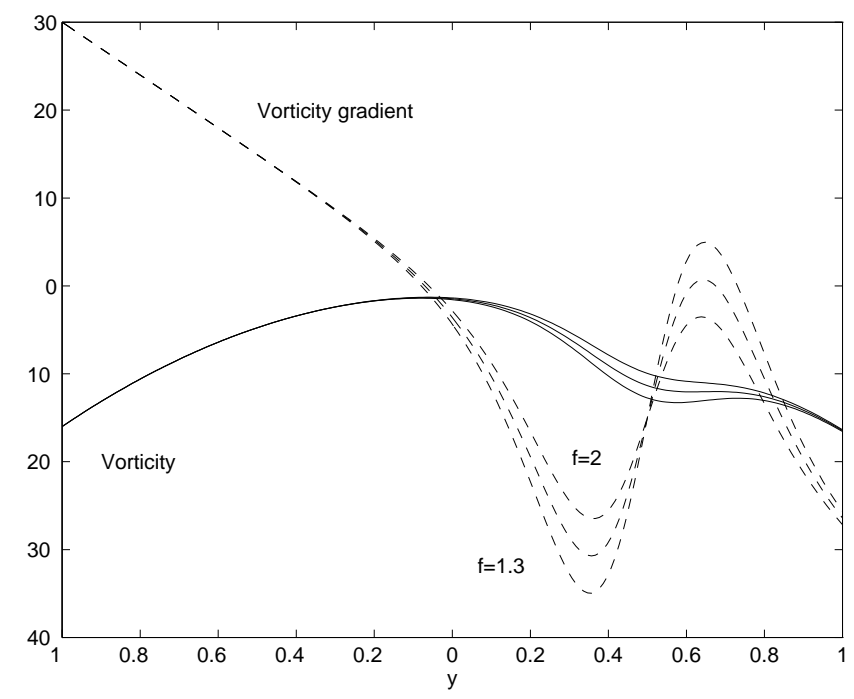

Figure 5: Vorticity and vorticity gradient profiles corresponding to the velocity profile $U(y)=$ $y+5 y^{3}+f \tanh 4(y-1 / 2)$ with equilibrium parameter values $f=1.3, f=1.65$, and $f=2$. At $f=f_{c} \approx 1.59$ two inflection points emerge.

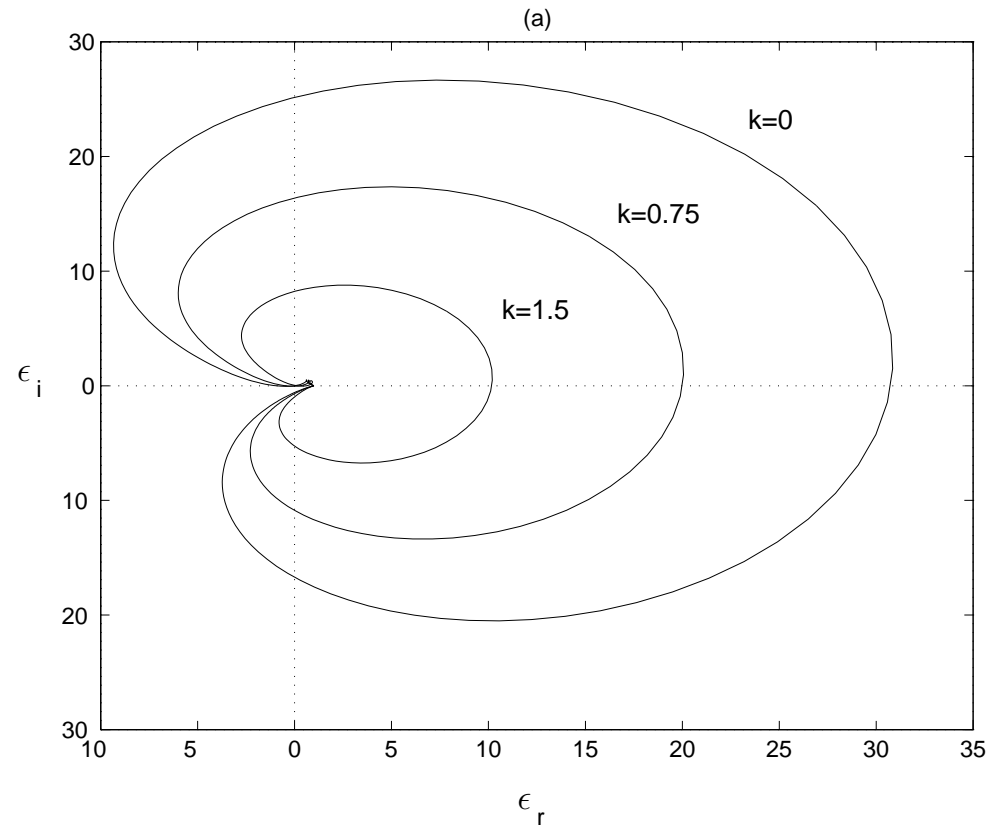




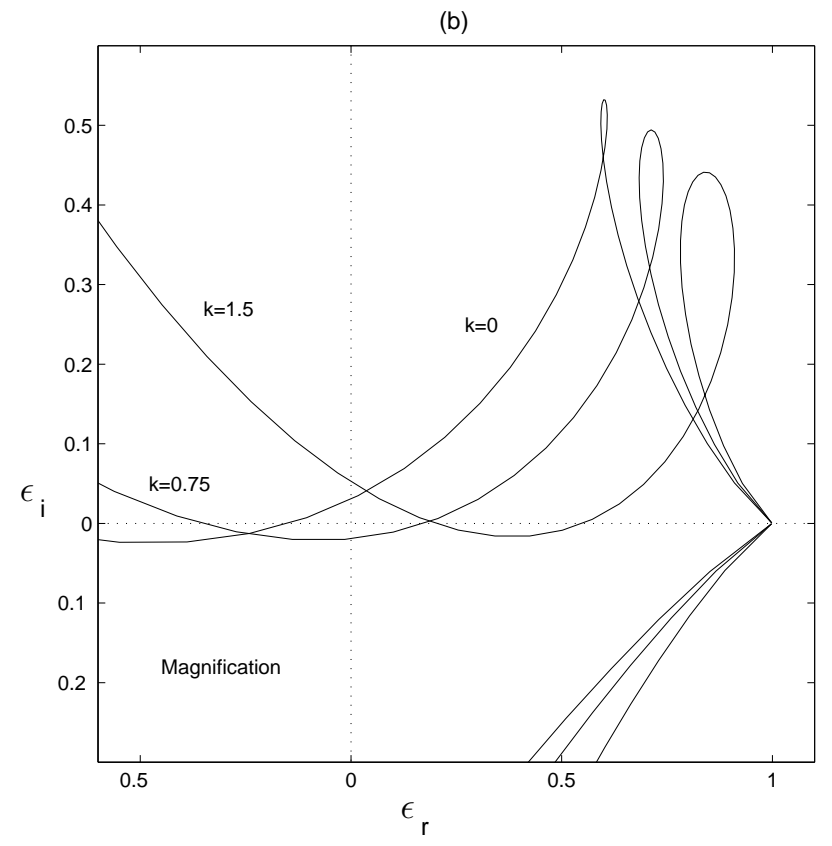

Figure 6: (a) Nyquist plots for the profile $U(y)=y+5 y^{3}+f \tanh 4(y-1 / 2)$ for $k=0,0.75$, and 1.5, and $f=1.62$. (b) Magnification that indicates the onset of instability through $k \neq 0$.

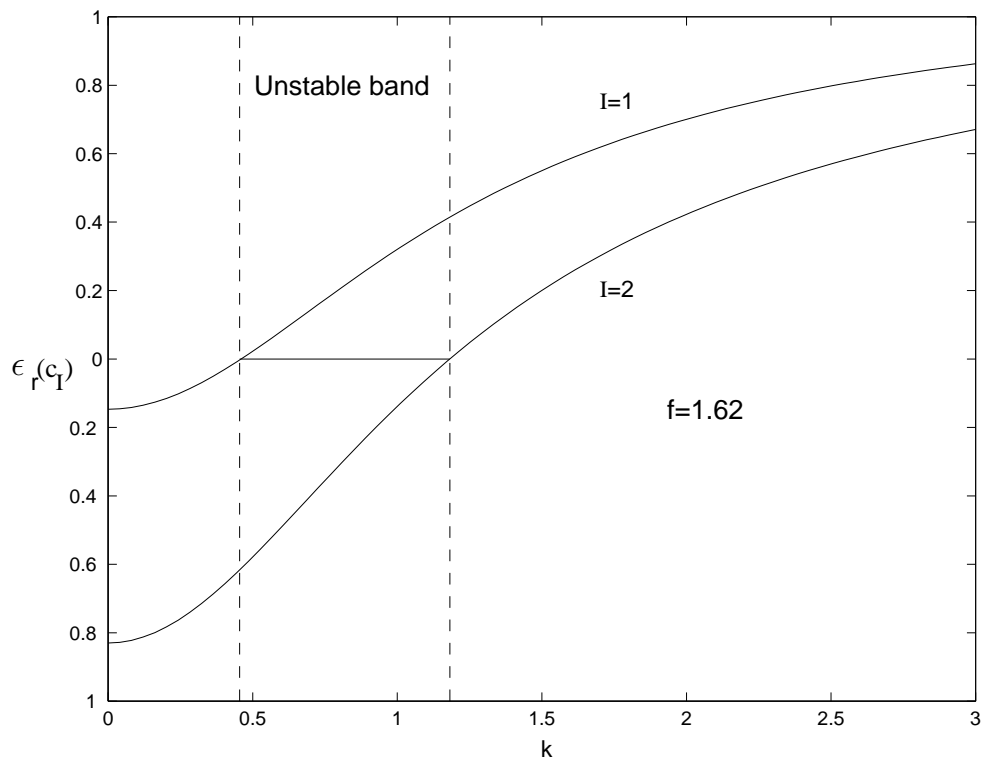

Figure 7: Plots of $\epsilon_{r}\left(c_{I}\right)$ against $k$ at $f=1.62$, where $c_{I}, I=1,2$, are the two inflection points leading to crossings of the Nyquist plot in Fig. 6 to the left of the asymptote $(1,0)$. 
variation of $\epsilon_{r}$ with $k$ for the two newly created inflection points at $f=1.62$; the range of unstable wavenumbers is $0.12 \lesssim k \lesssim 1.31$. Again the variation of $\epsilon_{r}$ with $k$ is monotonic.

The onset of the unstable band arises at the value of $f$ for which the two inflection points emerge; that is, this profile is the marginally stable state. Moreover, the critical value of $k$ is that required to make the nontranversal intersection of the path with the $\epsilon_{r}$-axis occur at the origin. Thus the emergence of the two inflection points as we raise $f$ leads to an instability with an onset at finite wavenumber, as shown in Fig. 3(c). Thus, in this case, instability is bounded away from $k=0$, in contrast to the previous examples. (This particular example is somewhat analogous to the bump-on-tail instability in the Vlasov problem [4]).

In both Figs. 4(d) and 7, $\epsilon_{r}$ appears to be a monotonically increasing function of $k$. If this feature were generally true, then the deformation of the Nyquist plots as we vary $k$ would certainly be simpler to understand. Indeed, this would imply that $\epsilon_{r}\left(c_{I}\right)<0$ evaluated at $k=0$ was sufficient for instability, independently of the number of inflection points (at least for monotonic, analytic profiles). However, we have found no argument implying such a property of $\epsilon_{r}$, and doubt one exists.

\section{Closing remarks}

We have presented in this paper a necessary and sufficient condition for the instability of monotonic shear flows. Several examples of equilibrium velocity profiles were treated in Sections 6 and 7 , demonstrating that the condition is of practical utility for finding instability and for understanding the kinds of bifurcations that can occur.

One may ask the question of how the method we have presented, which entails solving a Fredholm integral equation, compares with directly calculating the solutions numerically. Without the Nyquist method, one could solve Rayleigh's equation in order to locate unstable modes with a given $k$ for $c$ in the complex plane. This amounts to solving a boundary value problem with singular points. With this procedure one would repeatedly solve Rayleigh's equation at different values of (complex) $c$ and employ some kind of two-dimensional searching technique in order to find the eigenvalues. Whilst not especially difficult, this approach is computationally intensive, and in our opinion is neither elegant nor insightful. As a computational exercise, the calculation of the Nyquist function is substantially less effort than solving the boundary-value problem in this fashion. The Nyquist recipe is simpler because numerically it only requires a matrix inversion and only values of $c$ on the real axis are involved. Moreover, the sufficient condition of (50) requires one computation per inflection point, for each value of $k$, and no search in the complex plane.

In addition to these computational advantages, the Nyquist method provides insight into inviscid instability. It allows one to determine the number of unstable eigenmodes of a profile, as indicated, for example, by the multiple encircling of the origin of the Nyquist plots of Fig. 4. Also, it leads us to understand the various kinds of bifurcations to instability of a family of shear flow profiles. Put another way, Nyquist theory tells us how we can construct profiles with certain kinds of instabilities. For example, with the Nyquist imagery, we know how to build profiles that suffer the onset of instability at finite wavenumber, as in Section 7.3. Without this machinery, it would be much more difficult even formulating the problem of how to fashion the needed $U(y)$.

The bifurcations considered here are ones in which unstable eigenvalues appear out of a continuous spectrum. It is important to distinguish this kind of bifurcation from those that occur in systems with only discrete spectra, since any stable profile is arbitrarily close (in $L_{p}$ norm) to an 
unstable one (this is the essence of Gill's result [20] for Couette flow), a feature intimately linked with the presence of the continuous spectrum. It would then appear that catagorizing bifurcations to instability is problematic. However, the Nyquist plots in the $\left(\epsilon_{r}, \epsilon_{i}\right)$-plane provide a measure of how close a given equilibrium is from being deformed to one at the onset of instability. This onset may be through $k=0$ ( $c f$. Figs. 1 and 4 ) or at finite wavenumber ( $c f$. Fig. 6). Indeed, it can occur via more degenerate kinds of bifurcations, and these may be visualized straightforwardly with Nyquist theory. In fact, it is only the unclear dependence on $k$ that prevents us from immediately classifying every instability of a profile with a given number of inflection points (cf. [19]).

An example of the kind of insight provided by the Nyquist method is given by the following general result: for given $k$, in order to encircle the origin, the path must cross the $\epsilon_{r}$-axis twice. That is, in order to create a new instability we need two new inflection points. Thus, if there are $N$ inflection points in the profile (and so there are $N+1$ crossing of the $\epsilon_{r}$-axis altogether), there can be at most $(N+1) / 2$ unstable eigenmodes with that value of $k$. This result, which builds on a theorem of Howard [17], was stated without proof in [21].

In finite dimensional Hamiltonian systems bifurcations are regulated by Krein's theorem, which states that a necessary condition for the bifurcation to instability is that colliding eigenvalues possess energy signatures of opposite sign. It is also know for Hamiltonian systems that the constancy of energy can be used to obtain a sufficient but not necessary condition for stability. The results of this paper lend insight to and can be interpreted in the Hamiltonian context: the sufficient condition for stability based on energy is equivalent to the conditions of Rayleigh and Fjørtoft, and a version of Krein's theorem (and a notion of signature) for bifurcations involving the continuous spectra can be related to the Nyquist function [15].

We conclude with some remarks about the assumptions we made on the forms of the profile. The analytic structure of the Nyquist function heavily relies on the analytic form of the profile in the vicinity of $[-1,1]$. However, irrespective of the form of $U(y)$, we may nevertheless construct $\epsilon\left(c_{r}\right)$. In fact, the zeros of this function for $c_{r} \in[-1,1]$ still point to the existence of regular neutral eigenmodes even when $U(y)$ is not analytic. What is no longer clear is how to continue $\epsilon$ off the real axis. Or equivalently, whether there are discrete complex modes at nearby parameter values. Nevertheless there are various hints in this work that suggest that the theory works even if $U(y)$ has only two derivatives.

Finally, nonmonotonicity of $U(y)$ brings in the new complications of multiple critical layers for a given wave speed, and unusual properties of the streamfunction for critical layers lying at the shearless points where $U^{\prime}(y)=0$. It is for these reasons that the theory does not immediately carry over. We are currently working on these and other extensions.

\section{Acknowledgements}

This work was supported by the U.S. DoE under contract No. DE-FG05-80ET-53088. We thank J. B. Keller for commenting on the manuscript, and S. Friedlander for drawing our attention to reference [21].

\section{Appendix}

In this appendix we show that the change in the argument of the Wronskian is determined entirely by the piece of the contour $\mathcal{C}$ that is along the flow domain $(5 \rightarrow 7$ of Fig. 2$)$; the remainder of the 
contour will be shown to not encircle the origin.

The big semicircle: $1 \rightarrow 2 \rightarrow 3$.

On the big semicircle $c=R e^{i \theta}$ with $\theta \in(0, \pi)$. We consider the limit $R=|c| \rightarrow \infty$ by invoking the principle of permanence (see e.g. [22]), which in the present context simply states that the limit $c \rightarrow \infty$ of the solution to Rayleigh's equation at fixed $y$ is equal to the solution of the $c \rightarrow \infty$ limit of Rayleigh's equation. The latter limit is $\psi^{\prime \prime}-k^{2} \psi=0$, which has the following solution with the appropriate boundary conditions: $\Phi_{<}^{\infty}(y):=\sinh [k(y+1)] / k$. The principle of permanence implies $\Phi_{<}(y, c \rightarrow \infty)=\Phi_{<}^{\infty}(y)$, and thus using $W(c)=-\Phi_{<}(1, c)$ we obtain $\lim _{R \rightarrow \infty} W(c)=-\sinh (2 k) / k<0$. Therefore, the piece of the contour $1 \rightarrow 2 \rightarrow 3$ maps into the $W$-plane as a single point on the negative real axis (this is the asymptote $(1,0)$ on the $\epsilon$-plane).

As a check consider the limit $k \rightarrow 0$. Observe $\lim _{k \rightarrow 0} \Phi_{<}^{\infty}(1, c)=2$. (This is clearly correct since Rayleigh's equation becomes $\psi^{\prime \prime}=0$ and the assumed boundary conditions imply $\psi=1+y$, which when evaluated at $y=1$ gives 2.) Defining $F(c):=W(c) /\left[\left(U_{2}-c\right)\left(c-U_{1}\right)\right]$ gives $F(c, 0) \sim$ $2 e^{-2 i \theta} / R^{2}$, which is consistent with the $k=0$ result of [5].

The principle of permanence can be demonstrated explicitly by using the solution to Rayleigh's equation written as $\psi(y, c)=\sum_{n=0}^{\infty} \psi_{n}(y, c)$, where

$$
\psi_{n+1}(y, c)=\int_{-1}^{y}\left(y-y^{\prime}\right)\left(k^{2}+\frac{U^{\prime \prime}\left(y^{\prime}\right)}{U\left(y^{\prime}\right)-c}\right) \psi_{n}\left(y^{\prime}, c\right) d y^{\prime} .
$$

Using Cauchy's inequality, it is not difficult to prove that this series converges uniformly for all $y \neq y_{c}$. If we choose $\psi_{0}$ so that the boundary conditions of (2) are satisfied, evaluate (63) at $y=1$, and slip the $c \rightarrow \infty$ limit through the integral sign, then we obtain $\Phi_{<}^{\infty}(y)$.

As an aside, note that each $\psi_{n}$ is analytic in $c$. Thus because of uniform convergence, $\psi(1, c)$ is analytic for $c \neq U(1)$. This is true even for profiles $U(y)$ that are not analytically continuable into the complex plane; it is only necessary for the integral in (63) to exist to get analyticity in $c$. This puts a relatively mild restriction on $U$. For example, if $c$ is not in the flow domain, then $y U^{\prime \prime}(y) \in L_{1}[-1,1]$ is sufficient.

\section{The exterior legs: $3 \rightarrow 4$ and $8 \rightarrow 1$.}

We show below that if $c$ is not in the flow domain, which is the case on the legs $3 \rightarrow 4$ and $8 \rightarrow 1$ neither $\Phi_{<}(y, c)$ nor $\Phi_{>}(y, c)$ can vanish. Evaluating $\Phi_{<}$at $y=1$ we see the same is true for $W$. We also include a proof that $W$ is a monotonic functions of $c$ on $3 \rightarrow 4$ and $8 \rightarrow 1$. Thus these pieces of the contour map into curves that cannot cross into the right hand portion of the $W$-plane.

To prove the above statements we use a formula introduced by Green in the first half of the nineteenth century (see e.g. [22] or [10]). For Rayleigh's equation, Green's formula is

$$
\left[\psi\left(\psi^{\prime}-\frac{U^{\prime} \psi}{U-c}\right)\right]_{y_{0}}^{y}=\int_{y_{0}}^{y}\left[\left(\psi^{\prime}-\frac{U^{\prime} \psi}{U-c}\right)^{2}+k^{2} \psi^{2}\right] d y^{\prime}>0
$$

which is valid for any solution $\psi$. This formula can be derived from Rayleigh's equation by multiplying by $\psi$, manipulating, and integrating. It is important to remember that $c$ is assumed to be real and outside the domain of integration. 
Upon taking $y_{0}=-1, \psi=\Phi_{<}$, and applying the boundary condition $\Phi_{<}(-1, c)=0,(64)$ implies

$$
\Phi_{<}(y, c)\left[\Phi_{<}^{\prime}(y, c)-\frac{U^{\prime}(y) \Phi_{<}(y, c)}{U(y)-c}\right]>0,
$$

for all $y \in(-1,1]$ and $c \notin\left[U_{1}, U_{2}\right]$. This inequality means that neither factor can vanish for $y$ in the interior of the flow domain. We know that $\Phi_{<}(-1, c)=0$ and that $\Phi_{<}^{\prime}(-1, c)=1$, and therefore in a neighborhood of $y=-1$, by continuity of the solution, $\Phi_{<}(y, c)>0$. Thus $\Phi_{<}(y, c)>0$ for all $y \in(-1,1]$, and inequality (65) implies that the [ ]-factor must also be positive. (Note that neither factor can be singular by the existence theorem applied to Rayleigh's equation.) We mention, for later use, that a similar argument shows $\Phi_{>}(y, c)>0$.

Evaluating $\Phi_{<}$at $y=1$ yields $W(c)=-\Phi_{<}(1, c)<0$ for all $c \in\left(-\infty, U_{1}\right)$, which is our desired result for the leg $3 \rightarrow 4$. Similarly, $W(c)<0$ for all $c \in\left(U_{2}, \infty\right)$, our desired result for the leg $8 \rightarrow 1$.

We now further demonstrate that $W$ is monotonic, although this is not strictly needed for the proof. To this end we differentiate Rayleigh's equation with respect to $c$, yielding

$$
\frac{\partial \psi^{\prime \prime}}{\partial c}-\left(k^{2}+\frac{U^{\prime \prime}(y)}{U(y)-c}\right) \frac{\partial \psi}{\partial c}=\frac{U^{\prime \prime}(y)}{[U(y)-c]^{2}} \psi,
$$

which by the method of variation of parameters, is seen to have the following solution:

$$
\frac{\partial \psi(y, c)}{\partial c}=\int_{-1}^{y} \frac{\psi\left(y^{\prime}, c\right)}{W_{12}} \frac{U^{\prime \prime}\left(y^{\prime}\right)}{\left[U\left(y^{\prime}\right)-c\right]^{2}}\left[\psi_{1}\left(y^{\prime}, c\right) \psi_{2}(y, c)-\psi_{2}\left(y^{\prime}, c\right) \psi_{1}(y, c)\right] d y^{\prime} .
$$

Here $\psi_{1}$ and $\psi_{1}$ are any two independent solutions of Rayleigh's equation and

$$
W_{12}(c):=\left[\psi_{1}(y, c) \psi_{2}^{\prime}(y, c)-\psi_{2}(y, c) \psi_{1}^{\prime}(y, c)\right] .
$$

Observe that (67) satisfies $\partial \psi(-1, c) / \partial c=0$, which is consistent with $\psi(-1, c)=0$, and thus we may assume $\psi(y, c)=\Phi_{<}(y, c)$. Letting $\psi_{1}(y, c)=\Phi_{<}(y, c)$ and $\psi_{2}(y, c)=\Phi_{>}(y, c)$ gives

$$
W_{12}(c):=\left[\Phi_{<}(y, c) \Phi_{>}^{\prime}(y, c)-\Phi_{>}(y, c) \Phi_{<}^{\prime}(y, c)\right]=-\Phi_{>}(-1, c)=\Phi_{<}(1, c),
$$

and the expression (67) implies

$$
\frac{\partial \Phi_{<}(1, c)}{\partial c}=-\int_{-1}^{1} \frac{U^{\prime \prime}\left(y^{\prime}\right)}{\left[U\left(y^{\prime}\right)-c\right]^{2}} \Phi_{<}\left(y^{\prime}, c\right) \Phi_{>}\left(y^{\prime}, c\right) d y^{\prime}
$$

Integrating (70) by parts gives

$$
\begin{aligned}
\frac{\partial \Phi_{<}(1, c)}{\partial c} & =\int_{-1}^{1}\left\{\frac{-2 U^{\prime 2}}{\left[U\left(y^{\prime}\right)-c\right]^{3}} \Phi_{<}\left(y^{\prime}\right) \Phi_{>}\left(y^{\prime}\right)\right. \\
& \left.+\frac{U^{\prime}}{\left[U\left(y^{\prime}\right)-c\right]^{2}}\left[\Phi_{<}^{\prime}\left(y^{\prime}\right) \Phi_{>}\left(y^{\prime}\right)+\Phi_{<}\left(y^{\prime}\right) \Phi_{>}^{\prime}\left(y^{\prime}\right)\right]\right\} d y^{\prime},
\end{aligned}
$$

which upon insertion of (69) into its second term can be manipulated into

$$
\frac{\partial \Phi_{<}(1, c)}{\partial c}=K(c)+\mu(c) \Phi_{<}(1, c)
$$


where

$$
\mu(c):=\int_{-1}^{1} \frac{U^{\prime}\left(y^{\prime}\right)}{\left[U\left(y^{\prime}\right)-c\right]^{2}} d y^{\prime}=\frac{U_{2}-U_{1}}{\left(U_{1}-c\right)\left(U_{2}-c\right)}
$$

and

$$
K(c):=\int_{-1}^{1}\left\{\frac{2 U^{\prime}\left(y^{\prime}\right) \Phi_{>}\left(y^{\prime}\right)}{\left[U\left(y^{\prime}\right)-c\right]^{4}}\left[\Phi_{<}^{\prime}\left(y^{\prime}\right)-\frac{U^{\prime}\left(y^{\prime}\right) \Phi_{<}\left(y^{\prime}\right)}{U\left(y^{\prime}\right)-c}\right]\right\} d y^{\prime} .
$$

Clearly for $c \notin\left(U_{1}, U_{2}\right), \mu(c)>0$. The [ ]-factor of (74) is precisely the [ ]-factor of (65), which we showed is positive. Since $U^{\prime}(y)>0$ and $\Phi_{>}(y)>0$, for $y \in[-1,1)$, we see that $K(c)>0$. Thus we have established monotonicity: $\partial \Phi_{<}(1, c, k) / \partial c>0$ or $\partial W(c, k) / \partial c<0$ for $c \notin\left(U_{1}, U_{2}\right)$.

The boundary regions: $4 \rightarrow 5$ and $7 \rightarrow 8$.

Now we consider the pieces of the contour that skirt the boundaries of the flow domain. These are the pieces $4 \rightarrow 5$ and $7 \rightarrow 8$ of Fig. 2. We use the expressions $\psi_{g}$ and $\psi_{b}$ of (16) and (17) to construct $\Phi_{<}(y, c)$ according to the procedure described in Section 4. Upon enforcing the boundary conditions as described in that section, it is not difficult to show that the Wronskian has the following form:

$$
\begin{aligned}
W(c) & =-\Phi_{<}(1, c)=\chi_{1}(c)\left(U_{1}-c\right)+\chi_{2}(c)\left(U_{2}-c\right) \\
& +\left(U_{2}-c\right)\left(U_{1}-c\right)\left[\chi_{3}(c) \ln \left(U_{1}-c\right)+\chi_{4}(c) \ln \left(U_{2}-c\right)\right],
\end{aligned}
$$

where the functions $\chi_{i}$ for $i=1-4$ are analytic functions of $c$. The piece $4 \rightarrow 5$ can be pulled down into a little semicircle on which $c=U_{1}+\delta e^{i \theta}$, where $\delta>0$ and $\theta \in(-\pi, 0)$, which is still consistent with $c$ being in the upper half plane. Evidently, $\lim _{\delta \rightarrow 0} W(c)=\chi_{2}\left(U_{1}\right)\left(U_{2}-U_{1}\right)$. Since this piece of the contour must connect to the piece that terminates at 4 as $3 \rightarrow 4$, it follows that the piece of the contour $4 \rightarrow 5$ maps into a point on the negative real axis of the $W$-plane. Similarly, on $7 \rightarrow 8$, where $c=U_{2}+\delta e^{i \theta}, \delta>0$, and $\theta \in(-\pi, 0)$, we obtain $\lim _{\delta \rightarrow 0} W(c)=\chi_{1}\left(U_{2}\right)\left(U_{1}-U_{2}\right)$, and the piece of the contour $7 \rightarrow 8$ maps into a point on the negative real axis of the $W$-plane.

So in conclusion, since we have proven that for $c \in \mathcal{C}^{\prime}$ the real part of $W$ is negative, the only part of the contour that can give rise to a change in the argument of $W$ is the piece from $5 \rightarrow 7$.

\section{References}

[1] J. W. S. Rayleigh. On the stability or instability of certain fluid motions, Proc. Lond. Math. Soc. 9: 57-70 (1880).

[2] R. Fjørtoft. Application of integral theorems in deriving criteria of stability of laminar flow and for the baroclinic circular vortex, Geofys. Publ. 17: 1-52 (1950).

[3] E. M. Barston, On the linear stability of inviscid incompressible plane parallel flow, J. Fluid Mech. 233: 157-163 (1991).

[4] O. Penrose. Electrostatic instabilities of a uniform non-Maxwellian plasma, Phys. Fluids 3: 258-265 (1960).

[5] M. N. Rosenbluth and A. Simon. Necessary and sufficient conditions for the stability of plane parallel inviscid flow, Phys. Fluids 7: 557-558 (1964). 
[6] K. K. Tung. Barotropic Instability of Zonal Flows, J. Atmos. Sci. 38: 308-321 (1981).

[7] K. M. Case. Stability of inviscid plane Couette flow, Phys. Fluids 3: 143-148 (1960).

[8] P. G. Drazin and L. N. Howard. Hydrodynamic stability of parallel flow of inviscid fluid, Advances in Applied Mechanics 9: 1-89 (1966).

[9] S. I. Rosencrans and D. H. Sattinger. On the spectrum of an operator occurring in the theory of hydrodynamic stability, J. Math. and Phys. 45: 289-300 (1966).

[10] E. L. Ince. Ordinary Differential Equations (Dover, New York, 1944).

[11] E. T. Whittaker and G. N. Watson. A Course in Modern Analysis. 4th ed. (Cambridge Univ. Press, Cambridge, 1927).

[12] F. G. Tricomi. Integral Equations (Dover, New York, 1985).

[13] E. M. Barston. Electrostatic oscillations in inhomogeneous cold plasmas, Annals Phys. 29: 282-303 (1964).

[14] N. G. Van Kampen. On the theory of stationary waves in plasmas, Physica 21: 949-963 (1955).

[15] N. J. Balmforth and P. J. Morrison. Singular eigenfunctions for shearing fluids, preprint.

[16] F. D. Gakhov. Boundary Value Problems (Dover, New York, 1990).

[17] L. N. Howard. The number of unstable eigenmodes in hydrodynamic stability problems, J. de Méch. 3: 433-443 (1964).

[18] R. A. Smith and M. N. Rosenbluth. Algebraic instability of hollow electron columns and cylindrical vortices, Phys. Rev. Lett. 64: 649-652 (1990).

[19] N. J. Balmforth, D. del Castillo-Negrete and W. R. Young. Dynamics of vortical defects in shear, J. Fluid Mech. 333: 197-230 (1996).

[20] A. E. Gill. A mechanism for instability of plane Couette flow and of Poiseuille flow in a pipe, J. Fluid Mech. 21: 503-511 (1965).

[21] L. D. Fadeev. On the stability theory for stationary plane-parallel flows of ideal fluid Zapiski Nauchnykh Seminarov LOMI 21: 164-172 (1971).

[22] E. Hille. Ordinary Differential Equations in the Complex Domain. (Wiley, New York, 1976) pp. 37 and 73.

\author{
UNIVERSITY OF NOTTINGHAM \\ UNIVERSITY OF TEXAS AT AUSTIN
}




\section{Figure Captions:}

Figure 1: Nyquist plots for the single inflection point profile, $U(y)=\tanh \beta y$. (a) Four plots for $\beta=2$ and $k=0,1$, and 2 , and the critical value for the onset of instability, $k=k_{c} \simeq 1.832$ (dashed curve). (b) Four plots for $k=0$ and $\beta=1,1.5$, and 2, and the critical value for the onset of instability, $\beta=\beta_{c} \simeq 1.2$ (dashed curve).

Figure 2: The contours $\mathcal{C}, \mathcal{C}^{\prime}$, and $H$ in the $c$-plane. The closed contour $\mathcal{C}$ runs the entire circuit from $1 \rightarrow 2 \rightarrow \ldots \rightarrow 8 \rightarrow 1$, with the portion from $3 \rightarrow 1$ lifted infinitesimally above the real axis. The contour $\mathcal{C}^{\prime}$ is $\mathcal{C}$ with the piece along the flow domain, $5 \rightarrow 7$, removed. The contour $H$ (dashed) is Howard's semicircle, within which the unstable eigenvalues must lie.

Figure 3: Stability boundaries for the three equilibrium profiles of Sec. 7. Figures (a) and (b) depict the nucleation of instability through $k=0$, while (c) depicts the nucleation of instability through $k \neq 0$. In (b), the stability boundary of the second and third unstable modes is also shown.

Figure 4: Nyquist plots for the multiple inflection point profile, $U(y)=y+(\sin \gamma y) / 2 \gamma$, with $k=1$. (a) The case $\gamma=5$ has one encircling of the origin and thus one unstable eigenvalue. (b) In the case with $\gamma=7.5$ two more loops have appeared and the curve almost encircles the origin two more times. That is, it is close to the threshold of the instability of two further modes. (c) The case $\gamma=10$ has three encirclings of the origin and three unstable eigenvalues. (d) Depiction of the monotonic dependence of the crossing values to the left of the asymptote $(1,0)$ as a function of $k$.

Figure 5: Vorticity and vorticity gradient profiles corresponding to the velocity profile $U(y)=$ $y+5 y^{3}+f \tanh 4(y-1 / 2)$ with equilibrium parameter values $f=1.3, f=1.65$, and $f=2$. At $f=f_{c} \approx 1.59$ two inflection points emerge.

Figure 6: (a) Nyquist plots for the profile $U(y)=y+5 y^{3}+f \tanh 4(y-1 / 2)$ for $k=0,0.75$, and 1.5, and $f=1.62$. (b) Magnification that indicates the onset of instability through $k \neq 0$.

Figure 7: Plots of $\epsilon_{r}\left(c_{I}\right)$ against $k$ at $f=1.62$, where $c_{I}, I=1,2$, are the two inflection points leading to crossings of the Nyquist plot in Fig. 6 to the left of the asymptote $(1,0)$. 